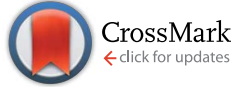

Cite this: Soft Matter, 2015, 11, 472

Received 7th September 2014 Accepted 16th October 2014

DOI: $10.1039 / c 4 s m 02007 c$

www.rsc.org/softmatter

\section{Kinetics of polymer looping with macromolecular crowding: effects of volume fraction and crowder size $\dagger$}

\begin{abstract}
Jaeoh Shin, ${ }^{a}$ Andrey G. Cherstvy ${ }^{a}$ and Ralf Metzler ${ }^{\star a b}$
The looping of polymers such as DNA is a fundamental process in the molecular biology of living cells, whose interior is characterised by a high degree of molecular crowding. We here investigate in detail the looping dynamics of flexible polymer chains in the presence of different degrees of crowding. From the analysis of the looping-unlooping rates and the looping probabilities of the chain ends we show that the presence of small crowders typically slows down the chain dynamics but larger crowders may in fact facilitate the looping. We rationalise these non-trivial and often counterintuitive effects of the crowder size on the looping kinetics in terms of an effective solution viscosity and standard excluded volume. It is shown that for small crowders the effect of an increased viscosity dominates, while for big crowders we argue that confinement effects (caging) prevail. The tradeoff between both trends can thus result in the impediment or facilitation of polymer looping, depending on the crowder size. We also examine how the crowding volume fraction, chain length, and the attraction strength of the contact groups of the polymer chain affect the looping kinetics and hairpin formation dynamics. Our results are relevant for DNA looping in the absence and presence of protein mediation, DNA hairpin formation, RNA folding, and the folding of polypeptide chains under biologically relevant high-crowding conditions.
\end{abstract}

\section{Introduction}

Molecular reactions in living biological cells are running off in a highly complex environment, that is compartmentalised by membrane structures and crowded with macromolecules and structural cytoskeletal networks. Macromolecular crowding (MMC) makes up a "superdense" environment modulating the kinetics of various biochemical processes in cells. Inter alia, this mechanism is employed biologically to tune the DNA accessibility in the cyto- and nucleoplasm. MMC non-trivially influences the levels of gene expression, and the size of the crowders dramatically modifies the response of genetic elements. ${ }^{2}$ In particular, it was found that in solutions of small crowders the rate of gene expression only varies slightly with the volume fraction $\phi$ of the crowders, while large crowders boost the expression levels many-fold. ${ }^{2}$

More specifically, MMC constitutes a non-specific environment controlling the looping properties of biopolymers such as nucleic acids and polypeptides. Polymer looping is indeed a ubiquitous mechanism of DNA protection, compaction, and gene regulation in both bacteria and higher organisms. ${ }^{3}$ DNA

${ }^{a}$ Institute for Physics \& Astronomy, University of Potsdam, D-14476 Potsdam-Golm, Germany.E-mail: rmetzler@uni-potsdam.de

${ }^{b}$ Department of Physics, Tampere University of Technology, FI-33101 Tampere, Finland $\dagger$ Electronic supplementary information (ESI) available. See DOI: $10.1039 / \mathrm{c} 4 \mathrm{sm} 02007 \mathrm{c}$ looping is vital for the regulation of transcription and effects the robustness of bio-switches. ${ }^{4}$ The effects of MMC on kinetics of DNA looping are of paramount importance for the speed, efficiency, and precision of gene regulatory networks. ${ }^{2,5,6}$ Inspired by the impressive body of experimental evidence for the relevance of MMC on biochemical processes, we here scrutinise the key role of the crowder size for the kinetics and thermodynamics of polymer looping.

The quantitative study of the diffusion-limited encounter of the end monomers of a polymer chain in a mixture of crowders of varying sizes and the analysis of the effective viscosity of the solution are formidable theoretical problems. Despite the progress of the understanding of polymer looping and cyclisation under dilute solvent conditions by theoretical approaches $^{7-10}$ and by simulations, ${ }^{11-17}$ polymer looping in the presence of $\mathrm{MMC}^{\mathbf{1 8 - 2 0}}$ still poses a number of challenges, which are our main targets here.

It is known that MMC generally facilitates the association of proteins via volume exclusion effects and favours more compact states. ${ }^{21}$ Polymer looping, however, involves the diffusion of an extended and chain length-dependent fragment of the polymer in crowded solutions. This non-locality effect renders the trends of the inhibition or facilitation of polymer looping kinetics in the presence of MMC less intuitive. Looping is a fundamental dynamic property of polymers which can be directly probed by methods such as fluorescence energy transfer. ${ }^{22}$ A comprehensive theory of polymer looping under crowded conditions is not 
straightforward. We here employ extensive crowder-explicit simulations of polymer looping including a number of important physical and biochemical ingredients.

Polymer organisation in the presence of MMC and spatial confinement is a common theme in biophysics. ${ }^{23}$ It affects, for instance, the segregation of DNA rings in dividing bacteria cells $^{24,25}$ as well as the territorial organisation of DNA inside eukaryotic nuclei ${ }^{26}$ and bacteria. ${ }^{27}$ Of particular interest is polymer looping and knotting in MMC-dominated solvents. ${ }^{28-30}$ The highly crowded environments of real biological cells feature volume occupancies of up to $\phi \sim 30 \% .^{31,32}$ In vitro, concentrated solutions of naturally occurring proteins, globular and branched polymers (lysozyme, serum albumin, PEG, dextran, Ficoll, etc.) mimic MMC conditions in a more controlled environment. ${ }^{33,34}$ On top of MMC volume exclusion, the eukaryotic cytoskeleton forms a spatial mesh with a period of several tens of nm affecting the diffusion of cellular components.

Excluded-volume interactions by crowders favour molecular association reactions,$^{35}$ speed up the folding of proteins into their native structures, ${ }^{36-40}$ and facilitate the assembly of virus capsids. ${ }^{41}$ The effects of the crowder size were studied for polypeptide folding ${ }^{42}$ and protein fibrillisation. ${ }^{35}$ We note that apart from MMC in the cytosol of biological cells, crowding is also an important ingredient for the diffusional dynamics of embedded proteins and lipid molecules in biological membranes. ${ }^{43-45}$ Also note that the thermodynamics and the demixing transitions in the mixtures of colloidal particles and linear polymers have been explored, ${ }^{47}$ in particular in the limit of long polymers (the so-called "protein limit"). ${ }^{46}$

The biological relevance for the study of polymer looping is due to its central role in gene regulation, for instance, in the formation of DNA loops induced by transcription factor proteins such as Lac or $\lambda$ repressor. ${ }^{48-50}$ The inter-segmental protein jumps along DNA made possible via looping facilitates protein diffusion in DNA coils ${ }^{51,52}$ and affects MMC-mediated gene regulation..$^{2,5,53,54}$ Another example is the dynamics of the DNA chain itself on various levels of DNA structural organisation ranging from bare DNA, via chromatin fibres, to complex chromosomal filaments. ${ }^{3,55}$ We also mention protein ${ }^{-56}$ and RNA-folding ${ }^{57}$ reactions.

Experimentally, the effects of polymeric crowders on the opening-closing dynamics of ssDNA hairpins with complementary sticky ends ${ }^{58,59}$ were studied in detail. ${ }^{60}$ It was demonstrated in ref. 60 that ssDNA hairpin formation dynamics is dramatically slowed down in highly crowded solutions of dextran and PEG of varying molecular weights (MWs), MW 0.2-10 kDa. Also, the fraction of open hairpins gets reduced substantially by relatively large crowders, in contrast to low-MW solutions of sucrose. In the latter, the similarly slowed-down DNA hairpin dynamics due to a higher viscosity of the medium, the fraction of hairpins remained nearly constant with crowding. Note that the experimental setup of ref. 60 only allowed the measurement of the geometric average of looping-unlooping times $\tau_{\mathrm{K}}$. A separate measurement of looping $T_{1}$ and unlooping $T_{\mathrm{ul}}$ times of the cohesive chain ends as a function of MMC fraction $\phi$ was not feasible. The fraction of time the hairpins are in a looped state was also measured. ${ }^{60}$
Some effects of MMC on polymer looping were analysed recently. ${ }^{11,19}$ For instance, for implicit attractive depletion potentials between polymer segments (mimicking MMC) the polymer looping $\left(T_{1}\right)$ and unlooping $\left(T_{\mathrm{ul}}\right)$ times (see below) for $\phi$ $=0.15$ and fixed size of crowders were quantified by simulations. ${ }^{11}$ For long chains, the increase of the looping time $T_{1}$ obeys the scaling relation

$$
T_{1}(n) \sim n^{2 v+1} \sim n^{2.2}
$$

with the chain length $l=n \sigma \cdot{ }^{11}$ Here $\nu \approx 3 / 5$ is the Flory exponent. ${ }^{90}$ Relation (1) is indeed supported by polymer cyclisation theory. ${ }^{16}$ Experimentally, the rate of formation of DNA hairpins drops somewhat faster with the chain length, $T_{1}(n) \sim n^{2.6 \pm 0.3}$, probably due to excluded-volume effects. ${ }^{58}$ Moreover, it was predicted that due to a non-trivial interplay of the enhanced solution viscosity and polymer "crumpling" the looping time varies non-monotonically with $\phi .{ }^{11}$

In contrast, the unlooping time $T_{\mathrm{ul}}$ exhibits only a weak dependence on the chain length. ${ }^{11} \mathrm{~A}$ finite cohesive energy of polymer ends, $\varepsilon_{\mathrm{s}}>0$, gives rise to more extended "looped" periods and longer unlooping times. ${ }^{11}$ The looping time, the time separating the extended and looped states of the chain, becomes shorter due to "depletion-based crowding" for longer chains, i.e., more compact polymer states are favoured, effecting a slow-down of the unlooping dynamics. ${ }^{11}$

We here report results from extensive Langevin dynamics simulations of the looping of Rouse-like flexible polymers in solutions of explicit nearly hard-sphere crowders (see Fig. 1). We examine the effects of the crowding volume fraction $\phi$, the crowder diameter $d_{\mathrm{cr}}$, the stickiness $\varepsilon_{\mathrm{s}}$ of the end monomers, and the chain length $n \sigma$, where $\sigma$ is the monomer diameter. We showed recently ${ }^{25}$ that for two polymer rings under confinement and crowding conditions the contact properties have been nonmonotonic in the crowding fraction $\phi$. Here, we demonstrate that MMC has unexpected effects on the looping dynamics as

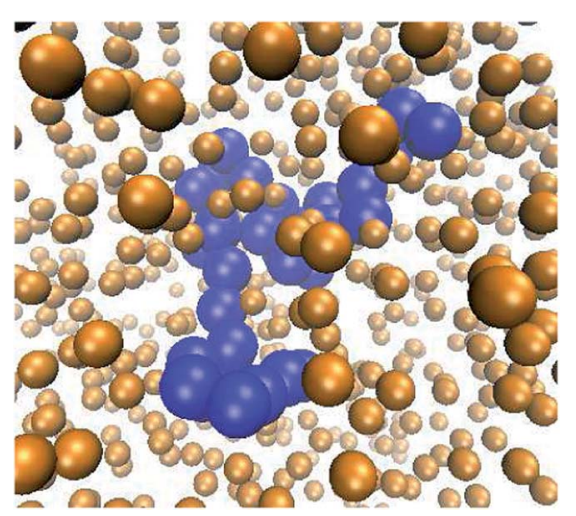

Fig. 1 Typical polymer conformation in the presence of MMC. The polymer chain (blue spheres) consists of $n=32$ monomers, the fraction of crowders (golden spheres, rendered smaller for better visibility of the polymer) is $\phi=0.1$, and the size of the crowders is $d_{\mathrm{cr}}=$ $1 \sigma$ in terms of the monomer diameter $\sigma$ of the polymer chain. Videofiles illustrating the looping dynamics of polymer chains for small and big crowders are included in the ESI.† 


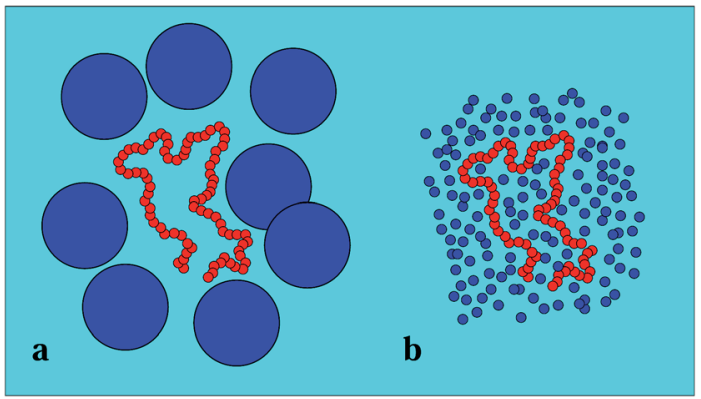

Fig. 2 Crowder size effect: large crowders lead to the caging of the polymer (a), while small crowders tend to mix with the chain monomers (b) and increase the effective viscosity. Note that the size of the chain is the same in both images.

well, due to the competition between depletion effects facilitating looping and an increased effective solution viscosity slowing down the looping kinetics, see Fig. 1 and 2.

\section{Model and methods}

To study polymer-nanoparticle mixtures by computer simulations, Monte-Carlo and molecular dynamics investigations were conducted in the literature to elucidate the static and dynamical behaviour of binary mixtures of polymers and crowders. Important ingredients were included in simulations to render the results applicable to realistic situations, for instance, in cells. Thus, the effects of compressible polymers, ${ }^{61}$ non-spherical ${ }^{62}$ and charged crowding nanoparticles ${ }^{63,64}$ on polymer-crowder demixing as well as the implications of confinement $t^{65,66}$ and viscoelastic effects $^{68}$ on polymer looping kinetics were studied.

Computer simulations ${ }^{11}$ revealed e.g. that the unlooping time $T_{\mathrm{ul}}$ remains nearly constant with chain length $n$ and increases 3-4 times as the crowding fraction grows from $\phi=$ 0 to 0.15 . Note that because of the limited applicability of the effective depletion potentials used, only moderate $\phi$ values were studied in ref. 11. The unlooping time $T_{\mathrm{ul}}$ is defined in our study as the time required for the chain to expand from the close-end to the equilibrium state, somewhat different from the definition used in ref. 11, see Fig. 3.

\section{A. Potentials and approximations}

Performing Langevin dynamics simulations of flexible polymers, we here examine the looping probabilities of the chain ends in the presence of MMC. The polymer chain is modelled within the bead-spring model with finitely extensible nonlinear elastic (FENE) potentials,

$$
U_{\mathrm{FENE}}(r)=-\frac{k}{2} r_{\max }^{2} \log \left(1-\frac{r^{2}}{r_{\max }^{2}}\right) .
$$

Here $k$ is the spring constant and $r_{\max }$ is the maximum allowed separation between the neighbouring polymer monomers. Excluded-volume interactions between polymer segments are given by the standard truncated Lennard-Jones (LJ) repulsive potential (Weeks-Chandler-Andersen potential),

$$
U_{\mathrm{LJ}}(r, \varepsilon)= \begin{cases}4 \varepsilon\left[(\sigma / r)^{12}-(\sigma / r)^{6}\right]+\varepsilon, & r<r_{\text {cutoff }} \\ 0, & \text { otherwise }\end{cases}
$$

with $r_{\text {cutoff }}=2^{1 / 6} \sigma$. Here, $r$ is the monomer-monomer distance, $\sigma$ is the chain monomer diameter, and $\varepsilon$ is the strength of the potential. We set $k=30, r_{\max }=1.5$ (to minimise bond crossings ${ }^{67}$ of the chain), and $\varepsilon=1$ (with all the energies being measured in units of the thermal energy, $k_{\mathrm{B}} T$ ). Similar repulsive 6-12 LJ potentials parameterise the (chain monomer)-crowder and crowder-crowder interactions.

The chain monomer diameter is set in simulations to $\sigma=4$ $\mathrm{nm}$, determining the polymer thickness and its effective viscosity in the crowded solution, $\eta$. The diameter $d_{\text {cr }}$ of monodisperse hard-core repulsive crowding particles varies in simulations in the range $0.75 \leq d_{\mathrm{cr}} \leq 8 \sigma$. The mass density is kept
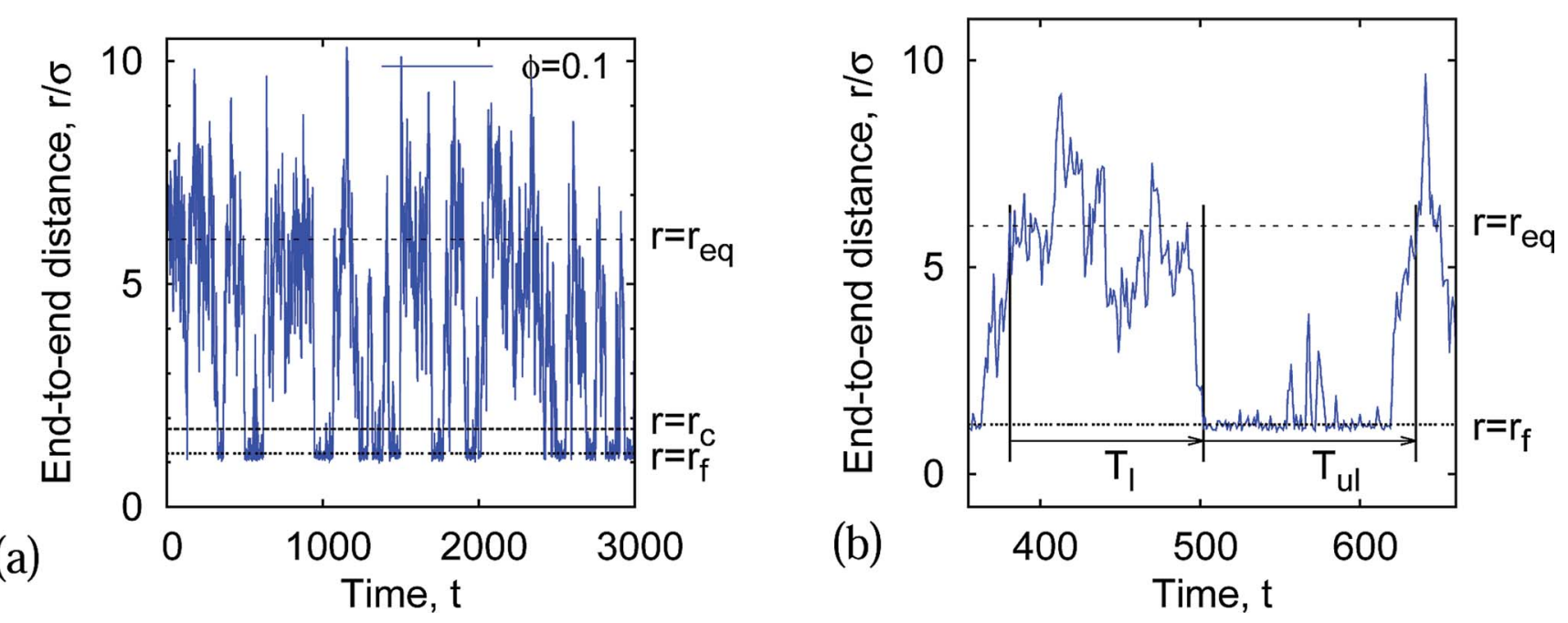

Fig. 3 Time evolution of the polymer end-to-end distance and definition of the looping-unlooping $T_{\mathrm{l}, \mathrm{ul}}$ and opening-closing $T_{\mathrm{op}, \mathrm{cl}}$. The equilibrium $r_{\text {eq }}$ and critical $r_{\mathrm{c}}$ distances are indicated. The simulated chain consists of $n=16$ monomers, and the crowding fraction is $\phi=0.1$. 
constant for all crowder sizes, fixed to the value known for average cytoplasm-crowding macromolecules. ${ }^{18}$ Thus, for the varying crowder sizes its mass grows as $m_{\mathrm{cr}} \sim d_{\mathrm{cr}}{ }^{3}$ and the friction coefficient increases according to the "effective" StokesEinstein law as $\xi_{\text {cr }} \sim d_{\text {cr }}$, similar to the procedure of ref. 76 . We use a cubic simulation box with volume $V=L^{3}$ and periodic boundary conditions. The volume fraction of crowders is $\phi=$ $N_{\mathrm{cr}} V_{\mathrm{cr}} / V$, where $N_{\mathrm{cr}}$ is the number of crowders and $V_{\mathrm{cr}}=\frac{4}{3} \pi\left(d_{\mathrm{cr}} / 2\right)^{3}$ the volume of each crowding particle. The characteristic time scale for a crowder with $d_{\mathrm{cr}}=1 \sigma$ and $m_{\mathrm{cr}}=$ $67.7 \mathrm{kDa}$ (ref. 18) is $\delta \tau=d_{\mathrm{cr}} \sqrt{m_{\mathrm{cr}} /\left(k_{\mathrm{B}} T\right)} \approx 0.36 \mathrm{~ns}$. The times presented in the figures below are in the units of this elementary time step $\delta \tau$. The features of the crowder size we observe with this explicit simulation scheme would not be visible in more coarse-grained models of crowded media employed previously, including those with effective depletion potentials.

The dynamics of the position $\mathbf{r}_{i}(t)$ of the chain monomers is described by the Langevin equation

$$
\begin{aligned}
m \frac{\mathrm{d}^{2} \mathbf{r}_{i}(t)}{\mathrm{d} t^{2}}= & -\sum_{j=1, j \neq 1}^{n} \boldsymbol{\nabla} U_{\mathrm{LJ}}\left(\left|\mathbf{r}_{i}-\mathbf{r}_{j}\right|\right)-\boldsymbol{\nabla} U_{\mathrm{FENE}}\left(\left|\mathbf{r}_{i}-\mathbf{r}_{i \pm 1}\right|\right) \\
& -\sum_{j=1}^{N_{\mathrm{cr}}} \boldsymbol{\nabla} U_{\mathrm{LJ}}\left(\left|\mathbf{r}_{i}-\mathbf{r}_{\mathrm{cr}, j}\right|\right)-\xi \mathbf{v}_{i}(t)+\mathbf{F}_{i}(t) .
\end{aligned}
$$

Here $m$ is the mass of the monomer, $\xi$ is the monomer friction coefficient and $\mathbf{F}_{i}(t)$ is white Gaussian noise with the correlator $\left\langle\mathbf{F}_{i}(t) \cdot \mathbf{F}_{j}\left(t^{\prime}\right)\right\rangle=6 \xi k_{\mathrm{B}} T \delta_{i j} \delta\left(t-t^{\prime}\right)$ that couples the particle friction and diffusivity $D=k_{\mathrm{B}} T / \xi$. Similar to the procedure described in ref. 69, we implement the velocity Verlet algorithm with the integration time step of $0.002 \leq \Delta t \leq 0.01$. A smaller simulation step was used for bigger crowders and higher volume fractions $\phi$.

The terminal monomers interact with the energy $\varepsilon_{\mathrm{s}}$ which mimics e.g. the energetic profit for the formation of closed ssDNA hairpin structures via hydrogen-bonding pairing interactions between the complementary bases on the end DNA fragments. Although we simulate flexible polymers, via corresponding rescaling of the effective monomer size, the results can be applicable to looping of semi-flexible dsDNA as well, where the loop/ring joining reaction is often supported by ligation enzymes. ${ }^{\mathbf{7 0 , 7 1}}$ The number of the chain monomers $n$ in the simulations varies in the range $10 \leq n \leq 256$. The pairing energy of $\varepsilon_{\mathrm{S}}=5 k_{\mathrm{B}} T$ used in the majority of results below can be considered as a good estimate for the pairing propensity in DNA hairpins with not too long complementary ends, see ref. 60 . We examine the range of chain end cohesiveness of $0 \leq \varepsilon_{\mathrm{s}} \leq 10 k_{\mathrm{B}} T$.

We simulate the attractive end-to-end interactions via the same LJ potential, eqn (3), but with a larger cutoff distance and bond intensity $\varepsilon_{\mathrm{s}}$, namely $U_{\mathrm{attr}}(r)=U_{\mathrm{LJ}}\left(r, \varepsilon_{\mathrm{s}}\right)+C_{\mathrm{LJ}}$ and $r_{\text {cutoff }}=$ $3 \sigma$. Along with this longer cutoff distance we shift the entire LJ potential in the vertical direction by the constant $C_{\mathrm{LJ}}$ so that at $r$ $=r_{\text {cutoff }}$ the potential becomes continuous with the zero-value branch at $r>r_{\text {cutoff. }}$ The volume fraction of mono-disperse crowders is varied in our simulations up to $\phi=0.3$; see ref. 72 for even denser colloidal systems.

The free energy of ssDNA hairpin formation contains two contributions: the favourable pairing of the helical dsDNA part and the entropic penalty of the looped part. The sum of the two for real DNAs is a complicated function of the DNA sequence and other model parameters ${ }^{73,75}$ amounting to $\sim-2.1 \mathrm{kcal}$ $\mathrm{mol}^{-1} \approx-3.5 k_{\mathrm{B}} T$ for about $20 \mathrm{bp}$ long DNA hairpins used in ref. 60. Longer complementary paired stem parts result in more stable hairpins, which we mimic in simulations via larger values of end-to-end cohesive energy $\varepsilon_{\mathrm{s}}$.

We neglect long-range interactions between polymer segments, including electrostatic forces, that is a reasonable approximation for long chains at physiological salt concentrations. In low-salt solutions, however, in application to DNA, the charge-charge interactions will become important for the loop-closure probability and dynamics. ${ }^{77}$ We assume that polymer-solvent interactions remain unaltered at increasing volume occupancies by crowders (see ref. 78 for possible effects of MMC on the properties of nucleic acid solutions at reduced solvent activity).

Hydrodynamic interactions are also neglected below (i.e., we use the Rouse polymer model), see ref. 79-81 for some implications. The effects of hydrodynamic interactions on endmonomers dynamics of dsDNA have been studied by fluorescence correlation spectroscopy experimentally in ref. 82 and 83 . Theoretically, the Rouse versus Zimm chain dynamics has been examined for semi-flexible polymers in solutions, ${ }^{\mathbf{8 4 , 8 5}}$ confined spaces, ${ }^{86}$ and near surfaces. ${ }^{87}$ In the latter situation e.g. it was clearly demonstrated, based on hydrodynamic Brownian simulations and mean-field hydrodynamic theory, how the Zimm dynamics turns into the Rouse one as the polymer chain approaches the no-slip surface. ${ }^{87}$ In particular, for the end-toend distance of the chain near the interface, the influence of hydrodynamic interactions screened as $\propto 1 / r$ with the interparticle distance was shown to be marginal.

\section{B. Parameters and data analysis}

We compute the end-joining statistics from the time series of the polymer end-to-end distance generated in simulations as follows. For looping, we start with the most probable end-to-end chain extension (the minimum of the free energy $F(r)$, see eqn (5) and Fig. 4, $r=r_{\text {eq }}$ ) and let the chain ends diffuse to the final extension $r=r_{\mathrm{f}} \approx 1.2 \sigma$. (The contact distance between the terminal chain beads in the folded state implemented in ref. 11 was somewhat different, $r_{\mathrm{f}}=\sigma+d_{\mathrm{cr}}$.) The looped state distance $r_{\mathrm{f}}$ corresponds to the minimum of the LJ potential in eqn (3) and remains nearly constant in the whole range of model parameters used here.

The time required for the chain to join its ends is defined as the looping time $T_{\mathrm{l}}$, see Fig. 3. The unlooping time $T_{\mathrm{ul}}$ is defined as the time required for the chain to expand back, from the jointed-ends state with $r=r_{\mathrm{f}}$ to the equilibrium state at $r=r_{\text {eq }}$.

In highly-crowded systems, which are the main targets of the current study, the polymer chain experiences collisions with many crowders around in the course of diffusion-limited looping. We thus believe hydrodynamic interactions to be of secondary importance for the static and dynamical effects considered here, likely just re-normalising the effective viscosity of the solution. As we demonstrate, rather the size of thermally-agitated crowders, which are to be displaced to ensure polymer looping, and their volume fraction are the dominant effects. 


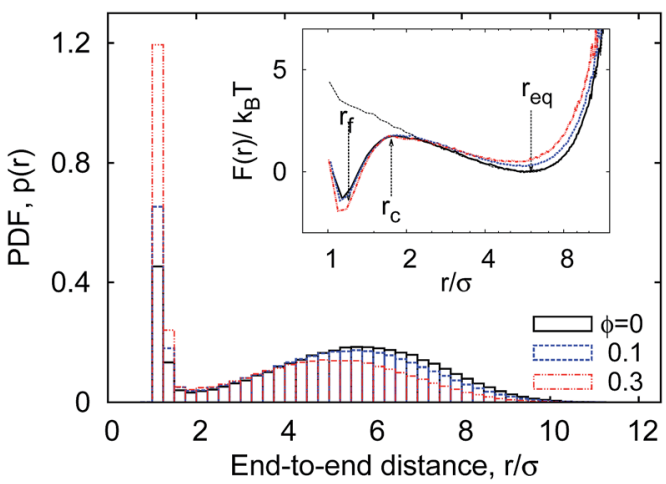

Fig. 4 Bimodal distribution $p(r)$ of the polymer end-to-end distance at varying MMC fraction $\phi$. The inset is the free energy profile for looping, $F(r)$, with the most likely separation between the polymer ends shown as $r_{\text {eq. }}$. The free energy profile for purely repulsive end monomers $\left(\varepsilon_{\mathrm{s}}=\right.$ $0, \phi=0)$ is also shown as the dotted curve in the inset. Parameters: $\varepsilon_{\mathrm{s}}=$ $5 k_{\mathrm{B}} T, n=16, d_{\mathrm{cr}}=1 \sigma$.

This distance is a function of all model parameters, in particular of the chain length $l=n \sigma$ and the MMC fraction, that is accounted for in simulations below. The closing time $T_{\mathrm{cl}}$ is defined as the average time the polymer needs to diffuse from the last moment its end-to-end extension was $r=r_{\mathrm{eq}}$ to the first moment with the close-contact distance of $r \approx r_{\mathrm{f}}$. The opening time $T_{\mathrm{op}}$ is the minimal time for the chain ends to diffuse from the closed state $r=r_{\mathrm{f}}$ to the first state with $r=r_{\text {eq. }}$. In Fig. 3 we illustrate on a real end-to-end diffusion trace the definitions of the looping/unlooping and opening/closing times.

Likewise, the critical distance of $r_{\mathrm{c}}=1.75 \sigma$ used below to define the occurrence of end-monomer contacts remains nearly constant. It approximately denotes the end-monomer separation at which the free energy barrier emerges which separates the close-looped and equilibrium states of the polymer, see Fig. 4. This critical distance $r_{\mathrm{c}}$ is used below to compute the looping probability $P_{1}$. One can think of other choices for $r_{\mathrm{c}}$ to mimic somewhat longer-ranged nature of end-end contacts.

We study the end-to-end joining statistics; the implications of MMC on looping kinetics of inner polymer monomers is beyond the scope of this study and will be presented elsewhere. The simulation time for the chains of $n=8,32$, and 128 monomers on a standard 3-3.5 GHz core machine is about 3, 4, and $60 \mathrm{~h}$, respectively. The typical number of the looping events used for an averaging procedure for these chain lengths is about 2000, 500, and 200 , correspondingly. In some cases we use traces, that are twice as long, for a better statistics. The number of crowding molecules of size $d_{\mathrm{cr}}=1 \sigma$ in the simulation box used to perform simulations of the polymer chains of these lengths is $N_{\mathrm{cr}} \approx 1000$, 3000 , and 10 000, respectively. Moreover, we remark that instead of averaging over the ensemble of initial chain configurations, we

$\S$ Note that starting from randomised chain configurations, in simulations of ref. 11 the looping time was computed till the chain ends are closer than a "critical" distance $r_{\mathrm{c}}$. The latter is an important parameter that depends on the type of interactions which act between the chain ends. It has a meaning of effective inter-segmental distance at which e.g. DNA-protein-DNA contacts can be established, $\approx 3-5 \mathrm{~nm}$ for a typical transcription factor. rather analyse the individual simulated time traces of the end-toend distance $r(t)$ to compute the chain looping characteristics.

We analysed the $r(t)$ data obtained from either single or multiple simulation runs, depending on the total computation time used. The typical running time, $t \sim 10^{5 \ldots 7} \times \delta \tau$, is chosen much longer than all the time scales in the system, in order to avoid a bias in sampling of end-joining events. To perform the error analysis, we use different methods for the dynamic and static quantities. As looping events are rare, the time intervals between them are of the order of the chain relaxation time, and the events can be considered independent. Thus, we use the standard error of the mean to compute the error bars for the looping (unlooping) and opening (closing) times. For the static quantities, such as the radius of gyration of the polymer, we split the entire trajectory into ten sub-series, calculate the values for each of them, and then compute the standard deviations of those pre-averaged values to get the final error bar. Previously ${ }^{25}$ we also used the so-called "blocking method" for the error analysis in correlated sets of data. Here we compare the two methods for a number of quantities and the differences in the sizes of the error bar were $\$ 30 \%$.

We need to distinguish the MMC effects for small $\left(d_{\text {cr }} \ll R_{\mathrm{g}}=\sqrt{\left\langle{R_{\mathrm{g}}}^{2}\right\rangle}\right)$ and large crowders $\left(d_{\mathrm{cr}} \gtrsim R_{\mathrm{g}}\right)$ as compared to the polymer's radius of gyration. Large crowders creates voids/cages between themselves which facilitate compaction of relatively short polymers and facilitate looping. The reader is referred to Section III G for the quantitative analysis of caging effects in our polymer-crowder mixtures. For longer chains, which do not fit into a single cavity and need to occupy the neighbouring voids, the effect of crowders on looping probability can be inverted. A similar effect occurs in MMC-mediated protein folding, when small crowders favour the compact state of a protein, while larger ones can promote protein unfolding. ${ }^{88}$ The systematic investigation of crowder surface properties is the subject of our future investigation. ${ }^{89}$

\section{Results: crowding and polymer dynamics}

The equilibrium statistical behaviour of a linear flexible polymers with sticky ends is governed by the tradeoff between the enthalpically favourable pairing of the sticky ends and the entropy loss in the more compact looped state. In what follows we first rationalise the effects of MMC on the static properties of polymer looping. We then examine the kinetics of loop closure and opening as functions of the details of the crowders such as the crowding fraction and crowder size.

\section{A. Distribution function $p(r)$ and free energy}

Our simulations generate time traces of the end-to-end distance $r(t)$ between the two extremities of the linear polymer. These two end monomers interact through an attractive $\mathrm{LJ}$ potential with cohesiveness $\varepsilon_{\mathrm{S}}$ which is varied in the range $0 \leq \varepsilon_{\mathrm{S}} \leq 10 k_{\mathrm{B}} T$, see the specification of the system in the preceding section. The recorded dynamics for $r(t)$ exhibits the highly erratic dynamics shown in Fig. 3. We first focus on the one-dimensional 

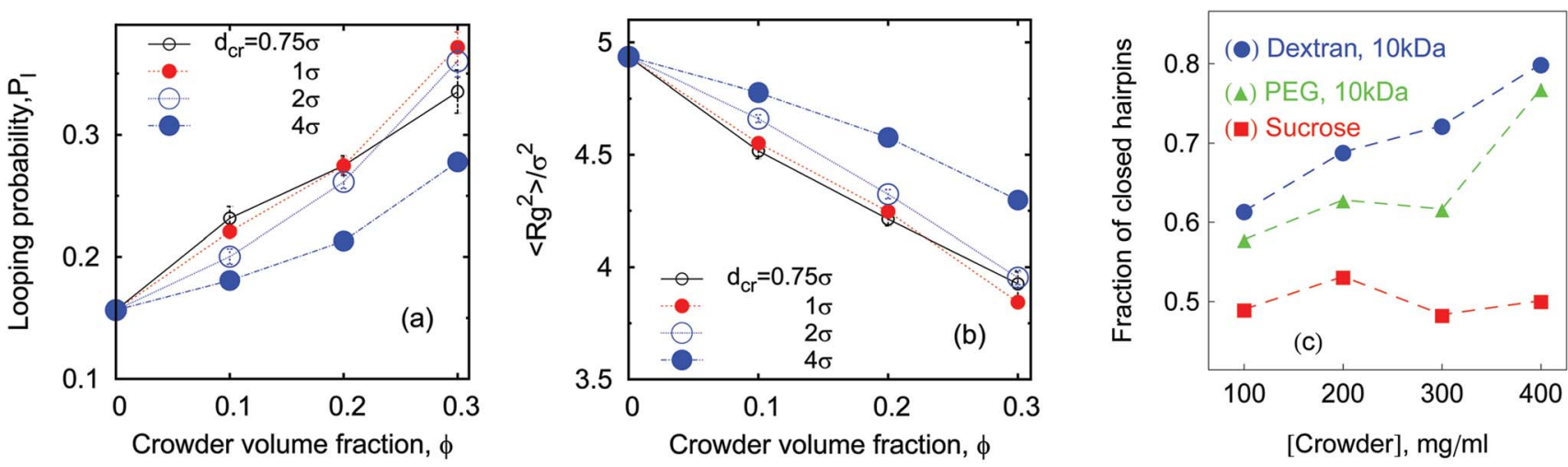

Fig. 5 Looping probability $P_{\mathrm{l}}(\phi)$ (panel a) and polymer radius of gyration $R_{\mathrm{g}}(\phi)$ (panel b) computed from simulations for $\varepsilon_{\mathrm{s}}=5 k_{\mathrm{B}} T, n=16$. Experimental data ${ }^{60}$ for the fraction of closed ssDNA hairpins is shown in panel (c). Here and below the simulation data for $d_{\mathrm{cr}}=1 \sigma$ are presented as small red circles, big blue circles correspond to $d_{\mathrm{cr}}=4 \sigma$. Note that the weaker effect of larger $\left(d_{\mathrm{cr}}=4 \sigma\right)$ crowders on the dimensions of the polymer coil shown in panel (b) may be due to the matching of sizes $\left(R_{\mathrm{g}} \sim d_{\mathrm{cr}}\right)$ for the relatively short chains considered here.

probability density function (PDF) $p(r)$ of the end-to-end distance, as shown in Fig. 4.

The relative motion of terminal monomers is subject to the free energy potential $F(r)$ that can be obtained from the PDF of the end-to-end distance $p(r)$ (see Fig. 4) via the inverse Boltzmann relation as

$$
F(r)=-k_{\mathrm{B}} T \log [p(r)]
$$

The presence of sticky chain ends gives rise to the formation of a double-well potential for $F(r)$, see Fig. 4 and 13. The shallow free energy well related to the maximum of the PDF $p(r)$ corresponds to the equilibrium end-to-end chain distance in the absence of sticky ends, namely $r=r_{\text {eq }}$. This minimum is accompanied by a sharp free energy well at very close end-to-end distances due to the presence of sticky ends. The transition between the looped and unlooped states of the polymer takes place in this asymmetric $F(r)$ potential. The chain should overcome free energy barriers in the course of looping and unlooping. Simultaneously, the equilibrium chain extension $r_{\mathrm{eq}}(n)$ is a growing function of the chain length, see Fig. 13.9

\section{B. Looping probability and polymer size}

From $p(r)$-which is a function of the number of monomers $n-$ we compute the probability

$$
P_{1}=\int_{\sigma}^{r_{\mathrm{c}}} p(r) \mathrm{d} r
$$

for the chain to be in the looped state as a function of $n$, see Fig. 13. That is, $P_{1}$ is proportional to the number of configurations in which the sticky ends of the chain are within a maximum distance of $r_{\mathrm{c}}=1.75 \sigma$. The lower cutoff discards thermodynamically unfavourable, rare events when the end beads are closer than the distance $\sigma$. For a single trajectory $r(t)$ of the end-to-end distance shown in Fig. 3 , the probability $P_{1}(n)$

I Note that the one-dimensional end-monomer distribution function $p(r)$ does not involve a Jacobian to recover the free energy profile $F(r)$ since our end-to-end distance measurements already account for the spatial dilation. is then equal to the fraction of time during which the chain is looped.

Fig. 5a demonstrates that the looping probability $P_{1}$ grows with the crowding fraction $\phi$. This is in accordance with the recent results of ssRNA tertiary folding-unfolding dynamics ${ }^{57}$ as well as ssDNA hairpin formation measurements ${ }^{60}$ in crowded polymeric solutions. In the latter experiment, fluorescence correlation spectroscopy data indicated a linear increase of the fraction of closed ssDNA hairpins as a function of $\phi$,

$$
P_{1}(\phi) \sim A+B \phi,
$$

see Fig. 5c. Our results reported here demonstrate that this trend becomes amplified for the growing length $n \sigma$ of the polymer, as demonstrated in Fig. 6a (compare the data sets for non-crowded and crowded systems). The magnitude of the relative facilitation for the looping probability for $\phi \approx 0.2$ is of the order of 2 to 4 , compared with the dynamics in the absence of crowders. This value is similar to the experimental trends for ssDNA hairpin formation with $\mathrm{MMC}^{60}$ compare Fig. 5c. This $P_{1}$-enhancement effect is present for both small and large crowders, as shown in Fig. 6a.

Consider now the PDF $p(r)$ shown in Fig. 4 . It has a bimodal structure, reflecting the proximity between the sticky ends with end-to-end distances $r \approx \sigma$ and a broad distribution of $r$ values
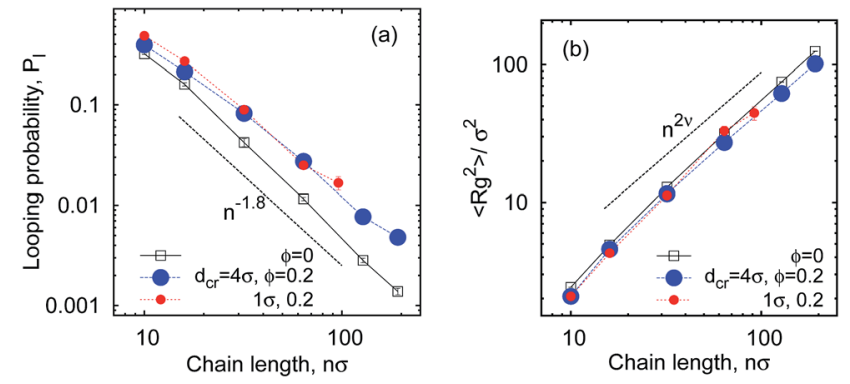

Fig. 6 Looping probability $P_{1}$ and the gyration radius $R_{\mathrm{g}}$ versus the degree of polymerisation $n$. The asymptotes $P_{\mathrm{l}}(n) \sim n^{-1.8}$ and $\left\langle R_{\mathrm{g}}{ }^{2}(n)\right\rangle$ $\sim n^{2 v}$ correspond to the dashed lines. Parameters: $\varepsilon_{\mathrm{s}}=5 k_{\mathrm{B}} T$ and $\phi=$ $0,0.2$. The crowder sizes are as indicated. 

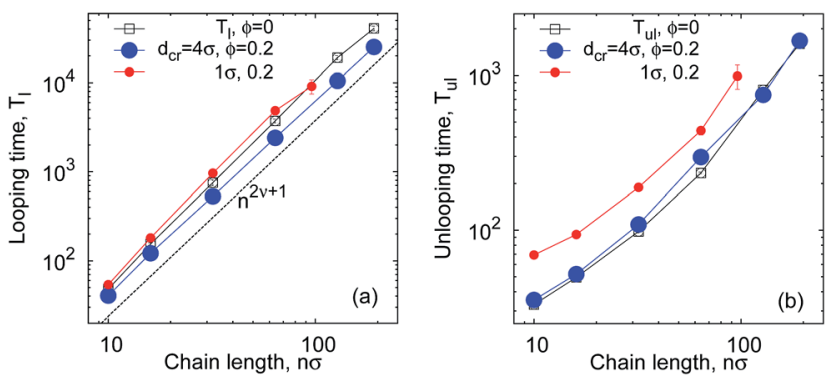

Fig. 7 Average looping (panel a) and unlooping (panel b) times versus the chain length. The asymptote (1) of $T_{1}(n) \sim n^{2 v+1}$ in the absence of crowders is shown by the dashed line in panel (a). Parameters are the same as in Fig. 6 . The shown error bars are often smaller than the symbol size.

reflecting the diffusive nature of the chain ends in the extended state. As can be seen in Fig. 4, the presence of MMC favours more compact polymer states: with increasing crowding fraction $\phi$ the polymer radius of gyration $R_{\mathrm{g}}$ decreases, in accordance with common MMC effects. ${ }^{21,56}$ The significant shift of the distribution to shorter $r$ values is particularly visible when the peak around $r \approx \sigma$ is considered.

For completeness we mention that, as expected a priori, stronger cohesiveness of the sticky ends favours higher looping probabilities $P_{1}$, reaching unity at $\varepsilon_{\mathrm{s}} \gg 1 k_{\mathrm{B}} T$ (see Fig. 14) and yields progressively longer unlooping times. This fact also agrees with the experimental data on ssDNA hairpin formation in solutions of polymeric crowders of different MWs shown in Fig. 8. Interestingly, we find that for crowder molecules with a larger diameter $d_{\mathrm{cr}}$ the looping probability $P_{1}$ becomes less sensitive to $\phi$, as demonstrated in Fig. 5a (smaller values of $B$ in eqn (7)). To map the detailed parametric dependence of the looping statistics as a function of chain length $n$, crowder size $d_{\mathrm{cr}}$, and fraction $\phi$ is a major challenge for simulations. We examine all these effects below.

As shown in Fig. 6 for both small and large crowders the looping probability $P_{1}$ decreases with the chain length $n$ as

$$
P_{1}(n) \sim n^{-1.8} .
$$

The scaling exponent 1.8 is close to the one of the Stockmayer formula $P_{1}(n) \simeq n^{-3 v}$ for the looping of a self-avoiding polymer, where $3 v \approx 1.76 .^{90}$ At the same time the radius of gyration of the chain grows as $\left\langle R_{g}{ }^{2}(n)\right\rangle \simeq n^{2 \nu}$, as expected for a self-avoiding chain. ${ }^{90}$ These dependencies are seen to be quite generic for varying crowder sizes $d_{\mathrm{cr}}$ and fractions $\phi$, see Fig. 6 .

The simulations yield instructive shapes for the polymer free energy $F(r)=-k_{\mathrm{B}} T \log [p(r)]$ as shown in the insets of Fig. 4 and in Fig. 13. We find a clear trend for the free energy barriers $\Delta F(n)$ : for the transition from the unlooped to the looped state the barriers become higher for longer chains, as shown in Fig. 13. This effect is due to the higher entropic penalty upon looping for longer polymers. In contrast, the barriers for a transition from the looped to the unlooped state are fairly insensitive to $n$, reflecting that unlooping is a local activation effect of dissolving the bond between the terminal monomers. This important feature gives rise to a more pronounced chainlength effect on the looping time $T_{1}$ as compared to the analogous dependence of the unlooping time $T_{\mathrm{ul}}$, as seen in Fig. 7. We now study the (un)looping times in more detail.

\section{Looping and unlooping times}

Fig. 3 shows how we extract the average looping and unlooping times $T_{1}$ and $T_{\mathrm{ul}}$ from the time series $r(t)$ of the end-to-end distance. Namely, $T_{1}$ is counted from the point when-after a previous looped state-the chain ends reach their equilibrium distance $r_{\mathrm{eq}}$ until they touch close to the minimum of the attractive LJ potential. By definition, the equilibrium distance $r_{\mathrm{eq}}$ corresponds to the free energy minimum for the extended chain conformations. From that moment, $T_{\mathrm{ul}}$ is counted until the chain ends are separated by the distance $r_{\mathrm{eq}}$ again. The computation of $T_{1}$ thus involves extensive chain rearrangements and thus non-trivially depends on the crowder fraction $\phi$, which favours more compact states. In our analysis $T_{1}$ and $T_{\mathrm{ul}}$ are then averaged over many looping events, the results being shown in Fig. 15 for a fixed chain length.

The distribution of looping times is found to be nearly exponential, and the characteristic time is shorter in more crowded solutions of bigger crowders, see Fig. 16. The full statistics and fluctuations of $T_{1}$ can be envisaged from the PDFs presented in Fig. 16. We fitted the $p\left(T_{1}\right)$ functions by two-parametric Weibull distributions of the form

$$
p\left(T_{1}\right) \sim T_{1}^{\gamma-1} \exp \left[-\left(T_{1} / T_{1}^{*}\right)^{\gamma}\right] .
$$

We found that the looping times are nearly exponentially distributed, with the parameter $1 \leq \gamma \leq 1.17$ being quite close to unity for all $\phi$ fractions and crowder sizes examined in Fig. 16. Note that the nearly - but not exactly-exponential distribution $p\left(T_{1}\right)$ is indicative of some short-living "intermediates" in the looping process. Note also that the first-encounter kinetics of the polymer ends is reminiscent of the first-passage kinetics of reactants in generalised biochemical networks, see e.g. ref. 91 and 92. The decay length $T_{1}^{*}$ of $p\left(T_{1}\right)$ distributions appears to be growing with $\phi$ for small crowders, while the decay of $p\left(T_{1}\right)$ gets faster with $\phi$ for larger crowders, see Fig. 16 for $d_{\mathrm{cr}}=1 \sigma$ and $4 \sigma$. This behaviour is physically consistent with the more restricted motions of the whole polymer and its ends at higher MMC fractions of bigger obstacles: the looping kinetics becomes faster and the spread of looping times gets narrower (more reliable statistics of looping events).

We also consider the opening and closing times $T_{\mathrm{op}}$ and $T_{\mathrm{cl}}$ (Fig. 3). $T_{\mathrm{op}}$ is the time for the chain ends to open up from a closed state and first reach the equilibrium distance $r_{\mathrm{eq}} \cdot T_{\mathrm{cl}}$ measures the time from the last occurrence of $r_{\mathrm{eq}}$ before a new looping event with $r<r_{\mathrm{c}}$. Both $T_{\mathrm{op}}$ and $T_{\mathrm{cl}}$ grow with $\phi$, as shown in Fig. 17. These times are, as expected, much shorter than the looping and unlooping times. For $T_{\mathrm{op}}$ and $T_{\mathrm{cl}}$ we detect no significant difference in their $\phi$ dependence, consistent with theoretical ${ }^{93}$ and experimental ${ }^{94}$ results.

So what about the dependence on the crowder size? Fig. 7 demonstrates that for small crowders the looping kinetics is somewhat inhibited and $T_{1}$ increases with $\phi$. For large crowders, however, we observe the opposite and stronger trend: polymer looping is facilitated. As detailed in Fig. 15a, $T_{1}$ indeed decreases with $\phi$ up to $d_{\mathrm{cr}}=4 \sigma$, however, for even larger crowders it starts to increase again, see Fig. 15b. For very large crowders $T_{1}$ appears to 

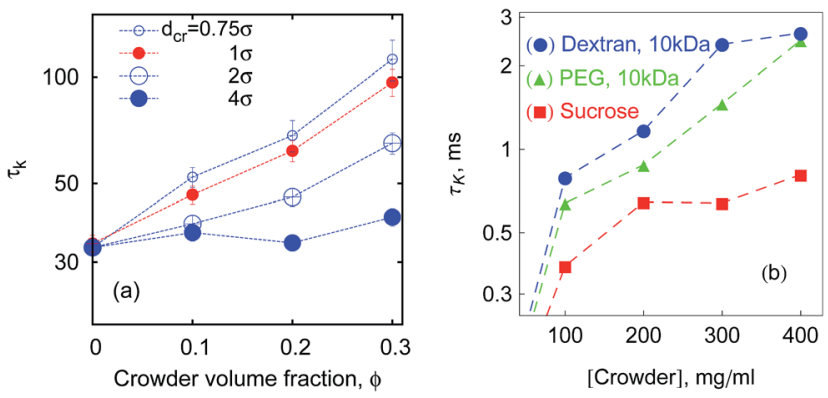

Fig. 8 Characteristic time $\tau_{\mathrm{K}}$ given by eqn (10) and computed from simulations (a) for $n=16$ and $\varepsilon_{\mathrm{s}}=5 k_{\mathrm{B}} T$. (b) Experimental data ${ }^{60}$ for ssDNA hairpin formation kinetics in solutions of different crowders. Both graphs are in log-linear scale.

approach the looping time in the absence of crowders, indicated by the dashed line in Fig. 15b. Fig. 18 reveals that the solution viscosity increases more strongly with $\phi$ for small crowders, slowing down the chain dynamics and reducing the looping rates. This non-trivial behaviour illustrated in Fig. 7 is our first key result.

Apart from the viscosity dependence, in Fig. 2 we highlight another important crowding-mediated effect. Namely, when the crowders are small, entropic effects favour a good mixing of crowders and chain monomers with little implications of the chain connectivity. When the crowders become larger, however, depletion effects become increasingly dominant. The chain becomes confined in a "cage". We emphasise here that the cage is not static but rather a dynamic entity, because of perpetual diffusion of crowders. Only at very high $\phi$ values or with possible attractions between the crowders the cage becomes static, as studied in ref. 74. In this confined state, the looping probability is significantly increased and thus the looping dynamics gets facilitated. As shown in Fig. 7 the depletion effect just outweighs the increased viscosity for larger crowders. The dynamics of crowders remains Brownian even at high volume fractions of $\phi \sim 0.3$, see below.

The unlooping time $T_{\mathrm{ul}}$, in contrast, typically increases with $\phi$. As shown in Fig. 7 and 15c, while the dependence of $T_{\mathrm{ul}}$ on $\phi$ is very weak for large crowders, it becomes quite sizable for smaller crowders. The effect on $T_{\mathrm{ul}}$ is due to both the higher viscosity induced by MMC and the impeded chain opening imposed by the caging effects. For the unlooping process both effects do not lead to an inversion of the $\phi$-dependence of $T_{\mathrm{ul}}$ inhibiting chain opening. The unlooping time is a monotonically decreasing function of the crowder size, see Fig. 15d.\|

\section{Comparison with DNA hairpin formation experiments}

Ref. 60 reports experimental data from fluorescence correlation measurements of ssDNA hairpin formation. The characteristic time $\tau_{\mathrm{K}}$ for the measured fluorescent blinking is given by the harmonic mean, ${ }^{60}$

\| The unlooping time is much shorter than the looping time and $T_{\mathrm{ul}}$ shows a weaker growth with $n$ than $T_{1}$, compare the two panels in Fig. 7. One possible reason is as follows. A looping event is the end-monomer encounter reaction that becomes progressively slower for larger polymer coils. The unlooping time in contrast is related to the (only moderately perturbed) diffusion of the polymer ends on the length-scale of the polymer coil, $r_{\text {eq }}(n) \sim R_{\mathrm{g}}(n)$.

$$
\tau_{\mathrm{K}}=T_{1} T_{\mathrm{ul}} /\left(T_{1}+T_{\mathrm{ul}}\right) .
$$

Similar to the experimental data, ${ }^{60}$ we show that $\tau_{\mathrm{K}}(\phi)$ has a tendency to grow with $\phi$ for crowders of all sizes and polymers of all lengths examined in the simulations, see Fig. 8 and 19. We observe that the typical variation of $\tau_{\mathrm{K}}$ with $\phi$ corresponds to a factor of $2-3$, in agreement with the measured data, ${ }^{60}$ as shown in Fig. 8. We also reveal a systematic dependence of the crowder diameter on $\tau_{\mathrm{K}}$ enhancement, in which smaller crowders are the most efficient, see Fig. 8. The curves in the plots indicate a nearly exponential dependence

$$
\tau_{\mathrm{K}}(\phi) \simeq \exp (\zeta \phi)
$$

as a function of the crowding fraction $\phi$. This is consistent with the exponential dependence of the self-diffusivity of a tracer in crowded solutions, $D(\phi) \sim \exp (-\zeta \phi) .{ }^{95}$ We checked that looping of longer polymers in crowded solutions yield qualitatively similar enhancement effects on $\tau_{\mathrm{K}}$ with $\phi$, see Fig. 19. In this figure the crowders are fairly large, $d_{\mathrm{cr}}=4 \sigma$, and the magnitude of $\tau_{\mathrm{K}}$ enhancement is somewhat smaller, consistent with the behaviour of $\tau_{\mathrm{K}}\left(d_{\mathrm{cr}}\right)$ presented in Fig. 8a.

For a quantitative comparison of our results for $\tau_{\mathrm{K}}$ to the experimentally observed $\tau_{\mathrm{K}}(\phi)$ enhancement, ${ }^{60}$ one needs to compare the relative sizes of polymers and crowders (experiment versus simulations). Namely, 10 kDa PEG polymers have $R_{\mathrm{g}, \mathrm{PEG}} \approx 2.8 \mathrm{~nm}$, while for 21-bp long DNA hairpins $R_{\mathrm{g}} \sim 7 \mathrm{~nm} .{ }^{60}$ In simulations, for $n=16$ chains, see Fig. 15 , the gyration radius is $R_{\mathrm{g}} \approx 2.5 \sigma$ (Fig. $5 \mathrm{~b}$ ), so the crowders of diameter $d_{\mathrm{cr}} \approx 2 \sigma$ are in the same relation to the polymer size in simulations as 21-bp DNA hairpins to $10 \mathrm{kDa}$ PEG in experiments. ${ }^{60}$ Note that $10 \mathrm{kDa}$ branched dextran polymers are smaller than $10 \mathrm{kDa} \mathrm{PEG}^{60}$ and the dynamics of DNA hairpin formation is slower in dextran solutions. The physical reason for this behaviour, as proposed in ref. 60, is a pronounced sub-diffusion of DNA hairpins in solutions of dextran with scaling exponent of $0.7<\beta<0.85$, in stark contrast to the sucrose and PEG solutions where the hairpin diffusion is nearly Brownian $(0.9<\beta<1)$, see also below.

\section{E. Length dependence and effective diffusivity}

The observed tradeoff between facilitation and inhibition is a fundamental feature of the looping kinetics for all chain lengths. Fig. 7 illustrates that the looping rate is systematically facilitated for larger crowders and impeded for smaller crowders. Concurrently, the scaling of the looping time with $n$ given by eqn (1) does not change appreciably in crowded solutions compared to the dilute case $\phi=0$, as shown in Fig. 7. This is our second important result.

Note that for longer polymers the accessible space inside the coil increases and at some point even large crowders can be accommodated therein, thus the reverting effect of polymer compaction by MMC. However, the gyration radius of our longest chains with $n \approx 200$ monomers is still too small to see this happen for the larger crowders $\left(d_{\mathrm{cr}}=4 \sigma\right)$ studied. Thus, the $T_{1}(n)$ scaling behaviour for even longer chains remains similar to the situation in the absence of crowders. We observe a slightly more pronounced looping time variation with $\phi$ for 


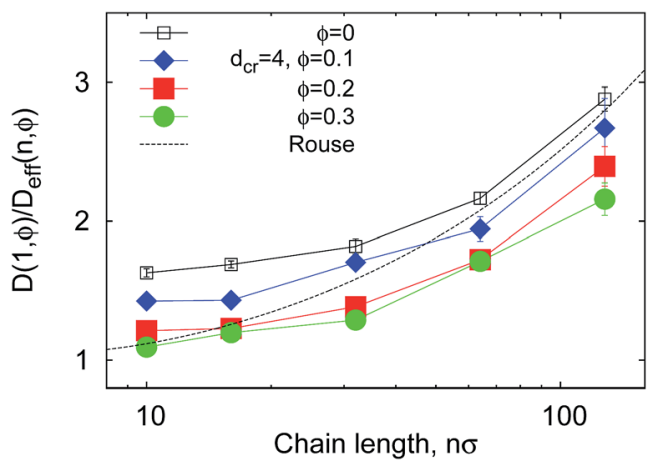

Fig. 9 Reciprocal effective diffusivity $D(1, \phi) / D_{\text {eff }}(n, \phi)$ of polymer ends, obtained from fit of the $T_{1}$ data in Fig. 7 with eqn (12). The Rouse chain result $D_{\mathrm{R}}(n, 0)$ given by eqn (13) with $D(1,0)=1 / 2$ as used in simulations is the dashed curve.

longer polymers in crowded solutions, in agreement with the well-established results, for instance, in protein-DNA interactions. ${ }^{2}$ The unlooping times vary substantially with the polymer length (in stark contrast to the observations of ref. 11). This indicates that unlooping is not a purely local unbinding process, but it needs the cooperative motion of the polymer.**

In Fig. 9 we study how many chain monomers are involved in looping events by quantifying the inverse effective positionindependent diffusivity $1 / D_{\text {eff }}$ of the end monomers. We use the data of Fig. 7 for $T_{1}$ and the general expression for mean firstpassage (i.e., looping) times

$$
T_{1}=\int_{r_{\mathrm{c}}}^{r_{\mathrm{eq}}} \mathrm{d} r^{\prime} \frac{\mathrm{e}^{F\left(r^{\prime}\right) /\left(k_{\mathrm{B}} T\right)}}{D_{\text {eff }}} \int_{r^{\prime}}^{n \sigma} \mathrm{d} r^{\prime \prime} \mathrm{e}^{-F\left(r^{\prime \prime}\right) /\left(k_{\mathrm{B}} T\right)},
$$

in a general potential $F(r) .{ }^{96}$ Here $n \sigma$ is the maximal chain extension. We fit the simulation data for $T_{1}$ with the free energy profiles $F(r)$ computed for each chain length in Fig. 13. The effective end-to-end diffusivity in the model of Rouse chains without crowding as derived in ref. 16,

$$
D_{\mathrm{R}}(n, 0) / D(1,0) \approx 8 / \sqrt{\pi n}-16 /(3 n),
$$

is represented by the dashed line in Fig. 9. Although our simulation data in the limit $n \gg 1$ follow this Rouse-chain prediction, the diffusivity of the terminal fragments in the presence of crowders for small $n$ shows sizeable deviations.

The effective number of monomers involved in the looping dynamics $n_{\text {eff }} \propto D(1, \phi) / D_{\text {eff }}(n, \phi)$ increases slightly with $n$ both for large and small crowders, as shown in Fig. 9. This figure illustrates that the number of chain monomers participating in looping slightly but systematically decreases with the MMC fraction $\phi$. The functional dependence of $D_{\text {eff }}$ is qualitatively similar to that of Rouse chains at larger $n$, see eqn (13), but with somewhat smaller $D_{\text {eff }}$ values. For smaller $n$, however, a plateau

\footnotetext{
** Such a statement is valid for relatively weak cohesion strength of the terminal monomers. In contrast, for very large end-to-end binding energies the unbinding kinetics is dominated by the dynamics of terminal monomers only, as illustrated by the Arrhenius-like behaviour for the unbinding events in Fig. 10. The motion of the polymer chain enables the accumulation of the energies $\gtrsim k_{\mathrm{B}} T$ required to disrupt the bond between the polymer ends.
}
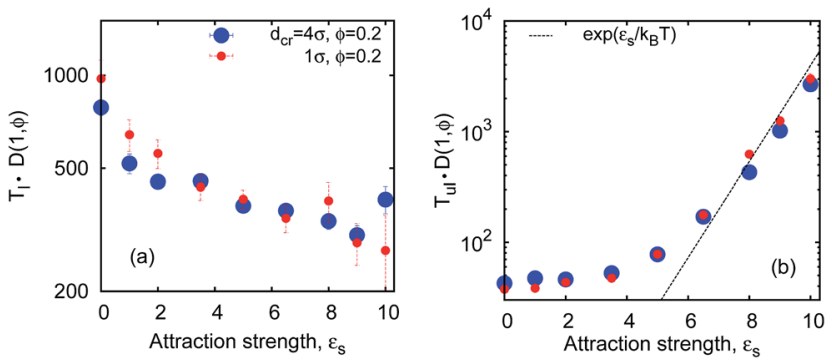

Fig. 10 Viscosity-renormalised looping (a) and unlooping times (b), namely $T_{\mathrm{l}, \mathrm{ul}} \rightarrow T_{\mathrm{l}, \mathrm{ul}} / \eta \sim T_{\mathrm{l}, \mathrm{ul}} D(1, \phi)$, at $\phi=0.2$ for varying $\varepsilon_{\mathrm{s}}$ and $n=32$. Note that with the increase of $\varepsilon_{\mathrm{s}}$ the number of looping events for the same trace length of $r(t)$ decreases, worsening the statistics.

of $D_{\text {eff }}$ is observed for all chain lengths in the simulations. For severe crowding we find that less monomers are involved in looping, compare the curves in Fig. 9. This analysis rationalises the cooperativity between the polymer extremities and the vicinal crowding particles. The $\phi$-dependent chain end diffusivity is our third main result. $\dagger \dagger$

\section{F. Effects of the binding affinity}

For ssDNA hairpins, the enthalpy gain of base-pairing upon looping is partly counter-balanced by the entropic penalty. ${ }^{104,105}$ For instance, for the 21-bp hairpin with CCCAA/GGGTT termini in ref. 60 the free energy of hairpin formation is $\sim 5 k_{\mathrm{B}} T .^{73}$ This value is used in our simulations for the end-to-end binding energy $\varepsilon_{\mathrm{s}}$, except for Fig. 10 where we vary $\varepsilon_{\mathrm{s}}$ in the broad range $0 \leq \varepsilon_{\mathrm{s}} \leq 10 k_{\mathrm{B}} T$. Larger $\varepsilon_{\mathrm{S}}$ values represent ssDNA hairpins with longer and thus more adhesive complementary end sequences. We observe a moderate, monotonic decrease of the looping time with $\varepsilon_{\mathrm{s}}$. Moreover, for all $\varepsilon_{\mathrm{s}}$ values longer looping times are obtained for smaller crowders and faster looping is detected for larger crowders (Fig. 10 and 20). This implies that our claims regarding the effects of crowding on the polymer looping dynamics are robust to changes of the model parameters.

Both looping and unlooping times can be rescaled by the effective solution viscosity $\eta \sim 1 / D(1, \phi)$ to yield universal dependencies for different crowder sizes as demonstrated in Fig. 10b. Fig. 20 shows the unscaled looping and unlooping data, together with the results for in the absence of crowding, revealing the same trends for $T_{1, \mathrm{ul}}$ with the crowder size as those presented for a fixed end-monomer affinity in Fig. 7b. The viscosity-based rescaling works particularly well for the

$\dagger$ Note that eqn (12) provides a satisfactory description of the looping times. ${ }^{12}$ Polymer looping is a prolonged barrier-crossing process in which the chain is close to equilibrium. For the reverse process of polymer unlooping, the disjoining of the end monomers takes place over a very short distance and spontaneous free-energy-downhill chain opening events occur (a process, which is inherently out of equilibrium). This is the main reason not to use the free energy-based eqn (12) to evaluate the times of chain unlooping. The latter consists of two terms, the time of disjoining the end monomers and their diffusion from a close distance to the separation $r_{\text {eq }}$. Depending on the attraction strength $\varepsilon_{\mathrm{s}}$, the relative contribution of the two terms to $T_{\mathrm{ul}}$ varies. For large $\varepsilon_{\mathrm{s}}$ values, for instance, the first contribution dominates so that the unlooping time exhibits the Arrhenius-like kinetics, see eqn (14) and Fig. 10. 
unlooping. As expected, for large end-to-end attraction $\varepsilon_{\mathrm{s}}$ the unlooping time grows and exhibits Arrhenius-like kinetics,

$$
T_{1}\left(\varepsilon_{\mathrm{s}}\right) \sim \exp \left[\varepsilon_{\mathrm{s}} /\left(k_{\mathrm{B}} T\right)\right],
$$

see the dotted line in Fig. 10b. These findings regarding the binding strength are our fourth key result. The exponential growth of the unlooping time with $\varepsilon_{\mathrm{s}}$ indicates the local physical nature of the unlooping process, in contrast to the looping kinetics at varying attractive strength $\varepsilon_{\mathrm{s}}$ which requires rather large-scale polymer re-organisations.

\section{G. Cavity and caging}

To quantify the already mentioned caging effects imposed by the crowders on the polymer coil, we explicitly compute the distribution of crowders around the polymer, as illustrated in Fig. 21. It shows that crowding particles of size comparable to the chain monomers diffuse quite substantially inside the coil volume. In contrast, crowders, whose sizes are much larger than the polymer monomers, are essentially excluded/depleted from the volume occupied by the polymer, thus facilitating polymer compaction and looping. Here, the reader is also referred to the investigation of caging effects in colloidal glasses. ${ }^{74}$

We also evaluated the correlation characteristics of the number of contacts $m_{\text {cr-p }}(t)$ that the polymer chain establishes with the neighbouring crowders in the course of time, see Fig. 22 for relatively large crowders. We define the normalised auto-correlation function of polymer-crowders contacts as ${ }^{25}$

$$
\operatorname{ACF}(\Delta)=\frac{\left\langle m_{\mathrm{cr}-\mathrm{p}}(t+\Delta) m_{\mathrm{cr}-\mathrm{p}}(t)\right\rangle-\left\langle m_{\mathrm{cr}-\mathrm{p}}(t+\Delta)\right\rangle\left\langle m_{\mathrm{cr}-\mathrm{p}}(t)\right\rangle}{\left\langle m_{\mathrm{cr}-\mathrm{p}}(t)^{2}\right\rangle-\left\langle m_{\mathrm{cr}-\mathrm{p}}(t)\right\rangle^{2}},
$$

where the angular brackets denote averaging along the $m_{\mathrm{cr}-\mathrm{p}}(t)$ trace. The critical distance between the centres of polymer monomers and neighbouring crowders in the algorithm is set to $R_{\mathrm{c}}=\sigma / 2+d_{\mathrm{cr}}$, such that at most one crowder fits between a crowder and a polymer monomer in contact. We checked that the observed $\operatorname{ACF}(\Delta)$ decay length is only weakly sensitive to the chosen critical contact distance $R_{\mathrm{c}}$ (results not shown).

We observe that, after an initial fast decrease of the number of contacts established, the further decay of the correlation function becomes nearly exponential, $\operatorname{ACF}(\Delta) \sim \exp \left[-\Delta / T^{*}\right]$, see Fig. 22. The corresponding decay length $T^{*}$ increases for longer polymers, partly due to a larger number of overall contacts $m_{\mathrm{cr}-\mathrm{p}}$ established. The characteristic time scale $T^{*}$ we obtain here is substantially shorter than the polymer looping time $T_{1}$ under the same conditions, compare Fig. 7 and 22.tt

\#. The reason is as follows. The decay time characterising $\operatorname{ACF}(\Delta)$ is related to the dynamics of individual monomer-crowder contacts. The interplay of these local fluctuations defines the life-time of a cage mediated by larger crowders for the entire polymer. The looping time is, on the other hand, a target-search problem for the encounter reaction of the two polymer ends in the same small region of space. Being impeded by a topological polymer structure chain looping takes place typically on much longer time scales than $T^{*}$.
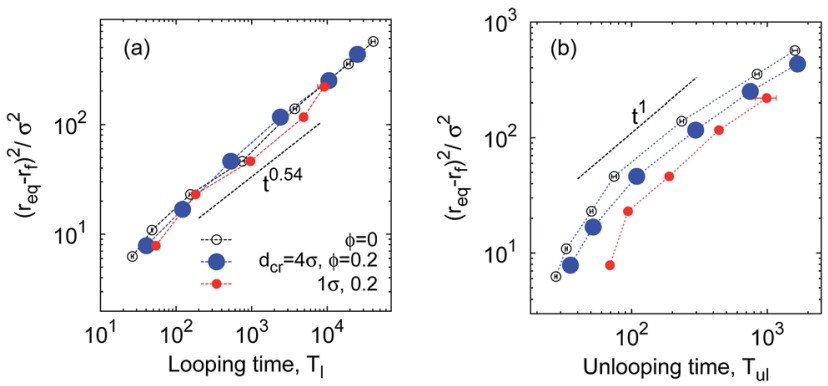

Fig. 11 Diffusion law of eqn (18) for looping and unlooping times of the chain ends necessary to bridge the distance $\left\langle\delta r(n)^{2}\right\rangle$. The asymptote of eqn (19) is the dotted line in panel (a); a linear scaling in panel (b) is included as a guide for the eye. Parameters are the same as in Fig. 7 and $\phi=0.2$.

\section{Results: diffusion}

\section{A. Subdiffusion of polymer ends}

MMC may impede the folding dynamics of short polypeptides due to a higher solution viscosity ${ }^{97}$ overwhelming the loopingfacilitating caging effects. A size-dependent diffusivity emerges: ${ }^{97}$ the diffusion of longer chains is impeded more strongly. Fig. 11 based on our simulations shows a similar effect for the mean squared looping distance versus the looping and unlooping times. This quantifies the diffusion law for looping events, i.e., the diffusive bridging of the distance

$$
\delta r=r_{\mathrm{eq}}-r_{\mathrm{c}}
$$

from the equilibrium distance $r_{\mathrm{eq}}$ of the sticky ends to the looped state with an end-to-end distance $r_{\mathrm{c}}$, and vice versa. As function of the chain length $n$, we checked that, similar to $R_{\mathrm{g}}$ in Fig. $6 \mathrm{~b}$, for longer polymers the scaling law

$$
\langle\delta r(n)\rangle^{2} \sim n^{2 v}
$$

is fulfilled. From the mean times $T_{1}$ and $T_{\mathrm{ul}}$ we compute the scaling exponents $\alpha$ from the generalised diffusion law ${ }^{98}$

$$
\left\langle\delta r(n)^{2}\right\rangle=2 D_{\alpha_{1}}\left\langle T_{1}(n)\right\rangle^{\alpha_{1}}=2 D_{\alpha_{\mathrm{ul}}}\left\langle T_{\mathrm{ul}}(n)\right\rangle^{\alpha_{\mathrm{ul}}} .
$$

Here $D_{\alpha_{i}}$ is the generalised diffusion coefficient in units of $\mathrm{cm}^{2}$ $\mathrm{s}^{-\alpha_{i}}$ and $\alpha_{i}$ the anomalous diffusion exponent for looping and unlooping processes, respectively. This approach helps us to distinguish the effects of the enhanced viscosity at higher $\phi$ from excluded-volume effects of crowders.

Fig. 11a illustrates that at large $\phi$ the looping dynamics is subdiffusive with $0.5 \leqq \alpha_{1} \lesssim 0.6$. This is but the standard result for polymer looping, as seen from combination of eqn (1) and (18),

$$
\left\langle\delta r(n)^{2}\right\rangle \sim T_{1}(n)^{2 \nu /(2 \nu+1)} \sim T_{1}(n)^{0.54}
$$

In contrast, for polymer unlooping no power-law scaling is found in the range of chain lengths $n \sigma$ considered here, see Fig. 11b. This fact is related to the absence of a power-law scaling in the $T_{\mathrm{ul}}(n)$ dependence, see Fig. $7 \mathrm{~b}$. 
These observations can be rationalised as follows. Once a thermal fluctuation breaks the bond between the sticky ends, the separation $r$ of the polymer ends drifts downhill in the free energy landscape $F(r)$ discussed above, quickly assuming larger values. In contrast, the looping time depends strongly on $n$ : to loop, the polymer needs to overcome an entropic penalty to get from $r_{\mathrm{eq}}$ to the contact distance $r_{\mathrm{c}}$, see Fig. 13. Thus, for looping it takes much longer to bridge the distance $\delta r(n)$ and involves interactions with a larger number of surrounding crowders, effecting the power law (19) with the small value $\alpha_{1}=0.54$.

\section{B. Diffusion of a tracer particle}

The size of the obstacles controls the facilitation or inhibition of polymer looping in crowded environments. Additionally, we exploit how fast the polymer ends join one another from the extended equilibrium state and reveal the regime of anomalous diffusion for the looping times with the scaling exponent of $\approx 0.54$, see eqn (19). Here we briefly examine whether this subdiffusive behaviour of extended polymer extremities is connected to any subdiffusion of a tracer particle in the crowded solutions simulated.

We compute the mean square displacement (MSD) for the diffusion of a single monomer of the chain (tracer particle), $\left\langle s^{2}(t)\right\rangle$, in crowded solutions with varying MMC fraction $\phi$ and crowder diameter $d_{\mathrm{cr}}$. Namely, we use the anomalous diffusion law $^{98-101}$

$$
\left\langle s^{2}(t)\right\rangle \sim t^{\beta}
$$

to compute the local scaling exponent

$$
\beta(t)=\mathrm{d}\left[\log \left(\left\langle s^{2}(t)\right\rangle\right)\right] / \mathrm{d}[\log (t)]
$$

along the ensemble averaged MSD trajectory. For Brownian motion $\beta(t) \equiv 1$ at all times.

We find that the viscosity of solutions of smaller crowders grows with $\phi$ faster than for larger obstacles, see Fig. 18. In this figure, the diffusivity has been extracted from the time averaged MSD along the $x$-direction,

$$
\overline{\delta_{x, i}{ }^{2}(\Delta)}=\frac{1}{t-\Delta} \int_{0}^{t-\Delta}\left[x_{i}\left(t^{\prime}+\Delta\right)-x_{i}\left(t^{\prime}\right)\right]^{2} \mathrm{~d} t^{\prime},
$$

in the lag time interval of $40<\Delta<400$, i.e., in the region where the linear scaling of the time average MSD is clearly established. Here $t$ denotes the total length of the tracer $x(t)$. This fast increase of the tracer's viscosity is consistent with the experimental measurements in crowded dextran solutions, see Fig. 5b in ref. 102. In the latter, the tracer exhibits an exponential growth of micro-viscosity with the concentration of polymeric crowders, valid for a wide range of relative tracer-crowder dimensions. The growth of viscosity with MMC fraction $\phi$ is also in accordance with theoretical predictions. ${ }^{\mathbf{1 0 3}}$

The ensemble averaged and time averaged MSD $\left\langle\overline{\delta_{x}{ }^{2}(\Delta)}\right\rangle=N^{-1} \sum_{i=1}^{N} \overline{\delta_{x, i}{ }^{2}(\Delta)}$ traces are identical in the long-time

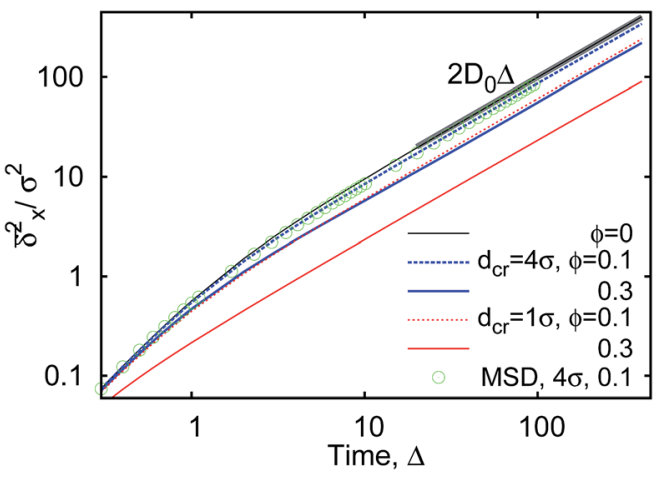

Fig. $12 \operatorname{MSD}\left\langle s^{2}(t)\right\rangle$ (green circles) and time averaged MSD $\left\langle\overline{\delta_{x}^{2}(\Delta)}\right\rangle$ along one spatial directions computed for varying crowder size and MMC fractions $\phi$ (as indicated in the plot). For each set of parameters, we average over $N=5$ time series for $\overline{\delta^{2}}$ and over $N=10^{3}$ traces for the MSD. The asymptote $M S D(t)=2 D t$ is the thick solid line, where $D=$ $D(1,0)$ is the single monomer free space diffusivity.

limit, see Fig. 12, with the long-time exponent $\beta$ being close to unity (Brownian motion). This indicates the ergodic tracer diffusion in the crowded solutions implemented in our simulations yields subdiffusive motion of the chain ends. In Fig. 12 we also show the ensemble and time averaged MSDs of a tracer particle with unit diameter in the crowded solutions. The diffusion exponent is nearly unity and no disparity of ensemble and time averaged displacements is detected, i.e., the motion is ergodic. ${ }^{99,101}$

\section{Discussion}

MMC non-specifically favours more compact conformations of proteins and speeds up their folding kinetics, ${ }^{21}$ as well as stabilises the proteins against thermal denaturation. ${ }^{95} \mathrm{MMC}$ may also reduce the occurrence of mis-folded states via reduction of the conformational space. ${ }^{38}$ The degree of crowding in living cells is heterogeneous and the crowders are polydisperse in size ${ }^{\mathbf{1 0 7}}$ giving rise to a micro-compartmentalisation of the cellular cytoplasm. ${ }^{\mathbf{1 0 6 , 1 0 8 , 1 0 9}}$ These effects pose the questions whether other fundamental elements of gene expression in biological cells are equally affected by MMC.

Specifically, recent gene-regulation experiments ${ }^{2}$ have shown that bigger dextran molecules increase the rates of gene expression by RNA polymerase to a higher-fold as compared to smaller ones. ${ }^{2}$ Bigger dextran molecules both reduce the diffusivity of RNA polymerase and enhance the number of binding events to the promoters (enhancing the association and reducing the dissociation rates). In solution of small crowders the impact of $\phi$ on gene expression rates is nonmonotonic (due to a compensation of moderate effects of MMC on the polymerase diffusivity and on its association rate to the DNA sites). In contrast, in solution of bigger crowders the expression rate grows monotonically and strongly with the $\phi$ fraction. $^{2}$ 
Here we show that indeed the looping kinetics of polymers such as DNA is highly sensitive to the volume fraction and size of crowders in a non-trivial way, and quantitative knowledge of this effect is necessary for the understanding of the molecular biological function of DNA based on looping. From extensive Langevin dynamics simulations we demonstrated that polymer looping is facilitated in the presence of large crowders, mainly due to depletion-based chain compaction. In contrast, for small crowders the dominant effect is the larger effective viscosity impeding the looping dynamics. The exact tradeoff between the two effects critically depends on the system parameters.

Our results are applicable to generic DNA looping and RNA folding dynamics in crowded systems, ${ }^{110}$ particularly, the formation of ssDNA hairpins with in vitro crowders. ${ }^{60}$ Here, our predictions for the crowder size and binding affinity effects can be tested directly in experiments. We already have showed that some predictions of our model indeed capture the experimental behaviour.$^{60}$ As targets for future studies, crowders of particular surface properties, non-inert poly-disperse and aspherical crowders will be studied..$^{89}$

In addition, the simulations of semi-flexible instead of flexible polymers in the presence of both MMC and external spherical confinement are expected to reveal a number of novel features. For instance, in contrast to free-space flexible chains studied here, the presence of spacial restrictions and finite bending energy penalty upon polymer looping yields a quasiperiodic but highly erratic dependence of the looping characteristics on the chain length $n \sigma$. Strong anti-correlation of the looping time and looping probability versus the polymerisation degree, pertinent for flexible chains, as those presented in Fig. $6 \mathrm{a}$ and $7 \mathrm{a}$, become more profound for the dynamics of cavity-confined semi-flexible polymers, see ref. 89 . We hope that our current investigation triggers new theoretical and experimental developments of static and dynamical properties of polymers in the crowded realm characteristic of the interior of living cells.\$§

$\S \S$ After submitting the current manuscript, we became aware of the recent studies of the crowder size. ${ }^{111,112}$ A stabilisation of intrinsically-disordered proteins and stabilisation of coil-to-globule transitions by crowding was discussed in ref. 111, based on computer simulations of an MMC-induced compaction of polymers. It was shown e.g. that smaller crowders exerting a higher osmotic pressure onto the polymer compact it to a larger extent, as compared to the bigger ones. In contrast to our observations, particularly small crowders are excluded from the space occupied by the self-avoiding polymer. Similar to our results, ref. 111 indicated that the size of the polymer coil reduces monotonically with $\phi$. A slight non-monotonic $R_{\mathrm{g}}(\phi)$ dependence obtained for the same system based on a phenomenological depletion potentials ${ }^{18}$ is thus rendered to be an artifact. ${ }^{111}$ The effects of MMC in our system are weaker than in ref. 111 (we have the Flory-like scaling of polymer dimensions and no coil-to-globule transitions occur). The difference may be due to a smaller size of crowders in ref. 111, as compared to the polymer monomers. Similar to our results presented in Fig. 5b, in ref. 113 smaller crowders were shown to be more efficient in compacting the polymer chain. Lastly, in ref. 112 the effects of the crowder size was investigated regarding the strength of depletion interactions between the two polymers. The strength of effective polymer-polymer attraction was shown to be reduced as the crowder size decreases (at a constant $\phi$ fraction).

\section{Appendix A}

In this Appendix we present the supplementary figures explaining the details of our main-text results.

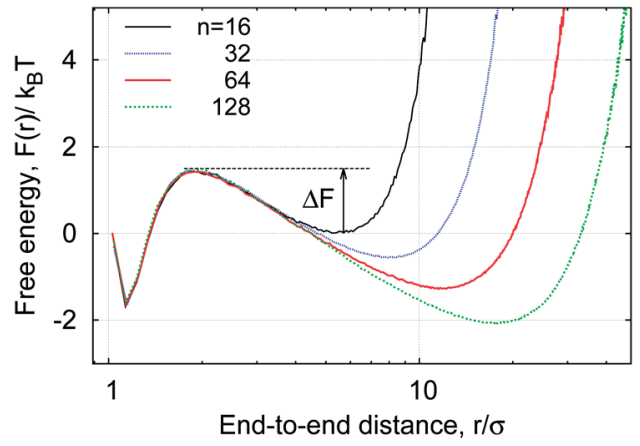

Fig. 13 The free energy landscape $F(r)$ for polymer looping for varying chain length at $\phi=0.2$ and $d_{\mathrm{cr}}=4 \sigma$. The energy minima at small endto-end distances are aligned in the plot in order to assess the barriers heights for looping, $\Delta F(n)$

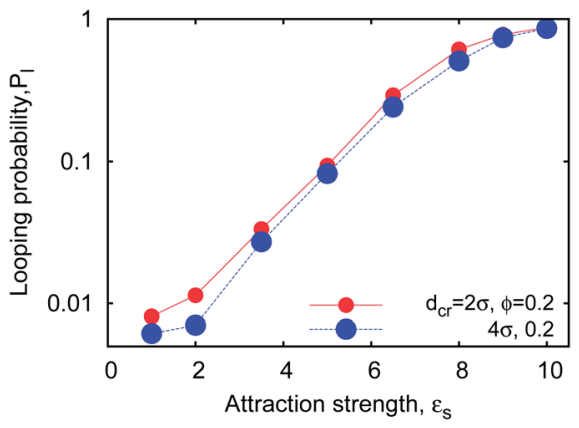

Fig. 14 Looping probability versus terminal monomer stickiness, computed for $n=32$ chains at different crowder sizes at $\phi=0.2$.
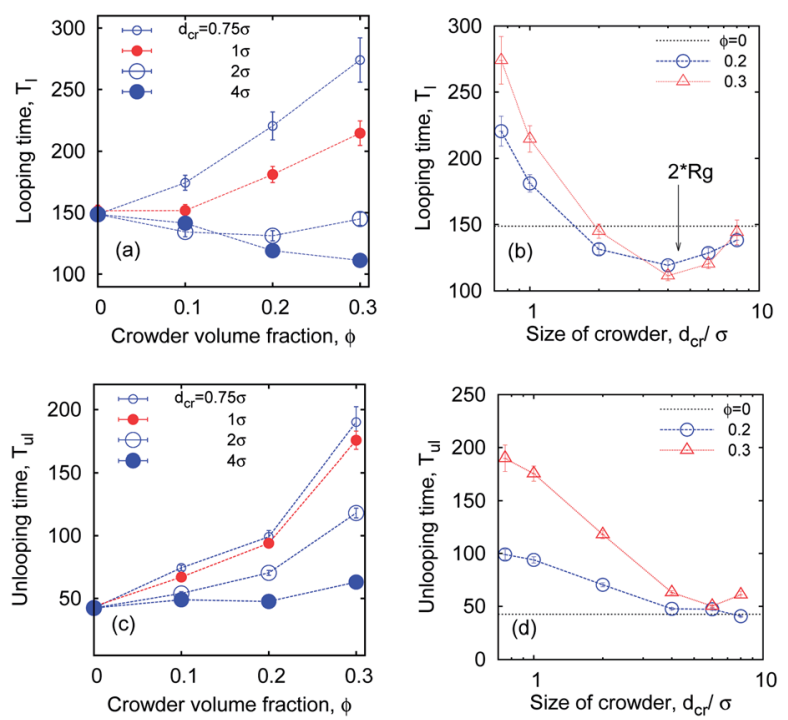

Fig. 15 Looping and unlooping times versus $\phi$, computed for a varying crowder size $d_{\mathrm{cr}}$, for short chains with $n=16$ monomers, and $\varepsilon_{\mathrm{s}}=5 k_{\mathrm{B}} T$. 

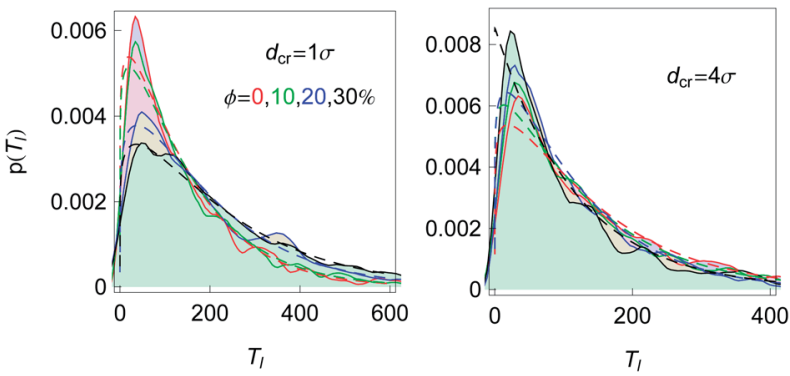

Fig. 16 The PDFs of looping times for $n=16, \varepsilon_{\mathrm{s}}=5 k_{\mathrm{B}} T$ and crowder sizes and fractions as indicated. Smoothed histograms are the simulations data and the dashed curves of the respective colour are the fits by eqn (9). The slight bumpiness of the histograms is due to the limited statistics of the generated looping events.

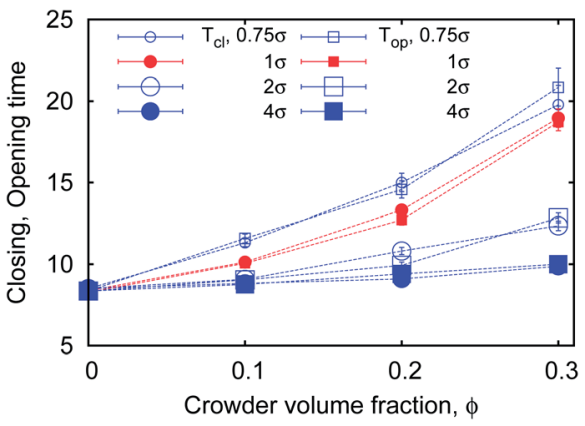

Fig. 17 Opening and closing times $T_{\text {op,cl }}$ versus $M M C$ fraction $\phi$ for varying crowder sizes. Parameters are the same as in Fig. 15.

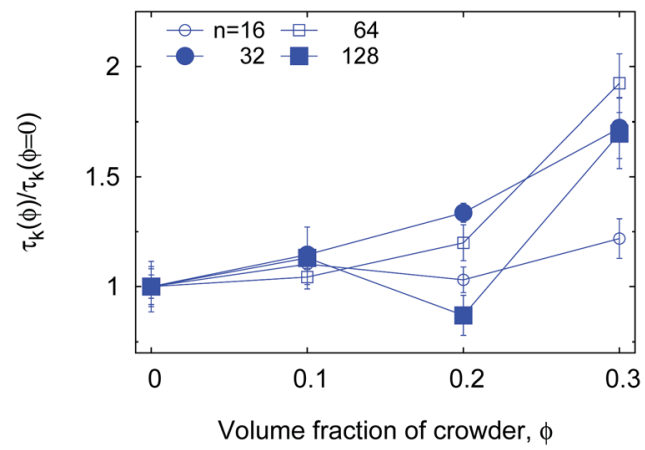

Fig. 19 The normalised $\tau_{\mathrm{K}}(\phi)$ for the varying chain length $n$, plotted for $\varepsilon_{\mathrm{s}}=5 k_{\mathrm{B}} T$ end-monomer adhesion strength and relatively big crowders $d_{\mathrm{cr}}=4 \sigma$. For small crowders the effect $\phi$ on the enhancement of $\tau_{\mathrm{K}}$ is stronger and more systematic (not shown due to significantly longer simulations times).
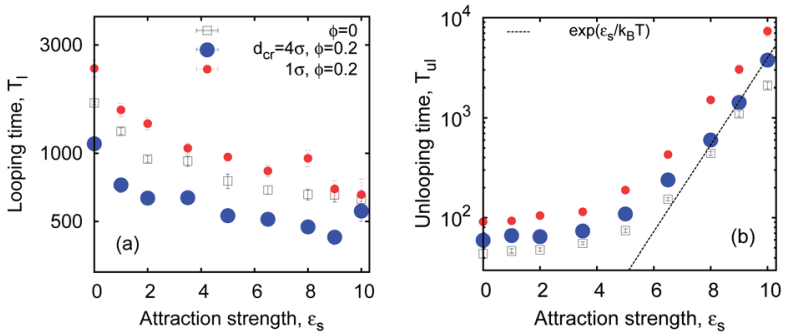

Fig. 20 The same data as in Fig. 10 but without normalisation by the effective viscosity of the solution, $\eta(\phi) \sim 1 / D(1, \phi)$. The data-set for the uncrowded solution is also included (open symbols).

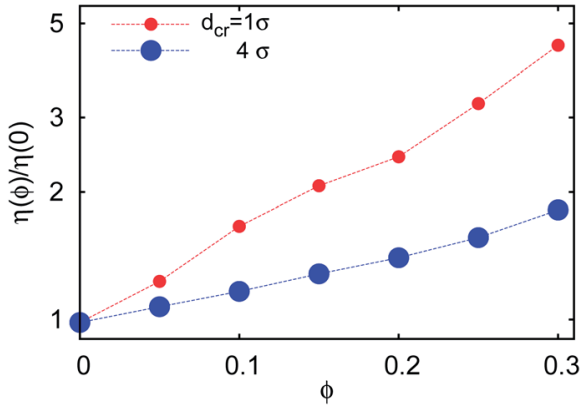

Fig. 18 Effective solution viscosity $\eta(\phi)=k_{\mathrm{B}} T /(3 \pi \sigma D(1, \phi))$ for a tracer of diameter $1 \sigma$ in solutions with varying crowder diameter $d_{c r}$ as extracted from the analysis of time averaged MSD traces.

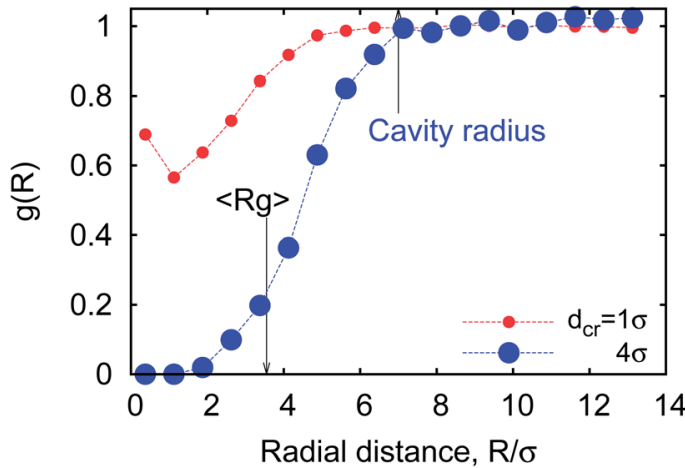

Fig. 21 The radial distribution function of relatively small (red dots) and large (blue dots) crowders around a polymer coil with $n=32$ monomers at MMC fraction of $\phi=0.1$. 


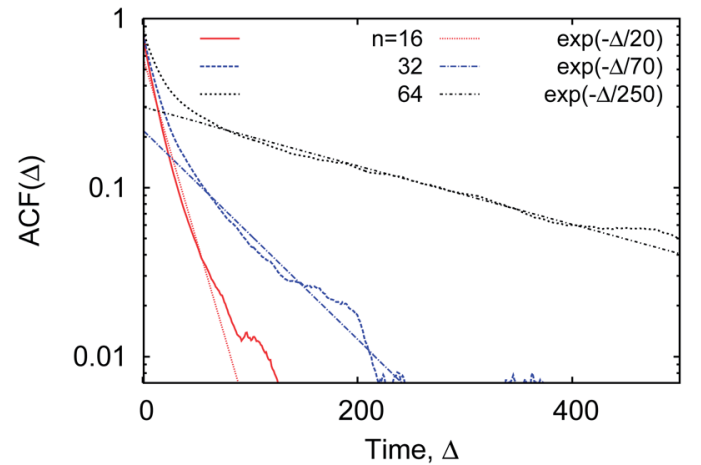

Fig. 22 The auto-correlation function of the polymer-crowders contact number (15), computed for polymers of varying length, at $\phi=$ 0.2 and $d_{c r}=4 \sigma$. The corresponding exponential asymptotes are shown as the dotted lines.

\section{Abbreviations}

$\begin{array}{ll}\text { MMC } & \text { Macromolecular crowding } \\ \text { PDF } & \text { Probability density function } \\ \text { LJ } & \text { Lennard-Jones } \\ \text { FENE } & \text { Finitely-extensible non-linear elastic } \\ \text { PEG } & \text { Polyethelene glycol } \\ \text { SSDNA } & \text { Single-stranded DNA } \\ \text { dsDNA } & \text { Double-stranded DNA } \\ \text { MW } & \text { Molecular weight } \\ \text { MSD } & \text { Mean squared displacement }\end{array}$

\section{Note from the authors}

After this paper was accepted, a number of relevant studies on this timely topic appeared. In particular, (i) the reader is referred to an extended review of Saxton [M. J. Saxton, J. Phys. Chem. B, 2014, 118, 12805] on the properties of various crowders used in vivo. (ii) The implications of crowders of varying size onto the asphericity of polymer chains in polymer-nanoparticle mixtures were examined by Monte-Carlo simulations by W. K. Lim and A. R. Denton, J. Chem. Phys., 2014, 141, 114909. (iii) The ring closure and loop formation reactions in the presence of reactive end groups of the polymer were examined by D. Sarkar, S. Thakur, Y. Tao and R. Kapral, Soft Matter, 2014, 10, 9577; there, the different scaling behaviour for the end-capture time with the polymer length was studied. (iv) Effects of loop stacking interactions and temperature on the thermodynamics of DNA hairpin formation were examined by M. Mosayebi et al., E-print arXiv:1410.1218. Similarly to our observations for the polymer looping time, the hairpin closing time was shown to be exponentially distributed. The variation of the hairpin closing time with temperature was shown to reveal a non-monotonic dependence, with a minimum close to the DNA melting temperature. This non-Arrhenius behavior is consistent with the experimental observations on DNA hairpin formation kinetics reported previously by M. I. Wallace, et al., Proc. Natl. Acad. Sci. USA, 2001, 98, 5584.

\section{Acknowledgements}

We thank D. Jost for discussions on hairpin formation and $\mathbf{M}$. Weiss for providing the experimental data for Fig. 5c and 8b. We acknowledge funding from the Academy of Finland (FiDiPro scheme to RM), the Deutsche Forschungsgemeinschaft (DFG Grant $\mathrm{CH} 707 / 5-1$ to AGC), and the Federal Ministry of Education and Research (BMBF Project to JS). We are particularly grateful to an anonymous referee for the insightful comments which improved our understanding of the subject.

\section{References}

1 I. Golding and E. C. Cox, Physical nature of bacterial cytoplasm, Phys. Rev. Lett., 2006, 96, 098102.

2 C. Tan, S. Saurabh, M. P. Bruchez, R. Schwartz and P. LeDuc, Molecular crowding shapes gene expression in synthetic cellular nanosystems, Nat. Nanotechnol., 2013, 8, 602-608.

3 R. Philips, J. Kondev, J. Theriot and H. Garcia, Physical Biology of the Cell, Garland Science, New York, 2012.

4 M. J. Morelli, P. R. ten Wolde and R. J. Allen, DNA looping provides stability and robustness to the bacteriophage $\lambda$ switch, Proc. Natl. Acad. Sci. U. S. A., 2009, 106(20), 81018106.

5 G. W. Li, O. G. Berg and J. Elf, Effects of macromolecular crowding and DNA looping on gene regulation kinetics, Nat. Phys., 2009, 5(4), 294-297.

6 H. Matsuda, G. G. Putzel, V. Backman and I. Szleifer, Macromolecular Crowding as a Regulator of Gene Transcription, Biophys. J., 2014, 106(4), 1801-1810.

7 G. Wilemski and M. Fixman, Diffusion-controlled intrachain reactions of polymers, J. Chem. Phys., 1974, 60(3), 866-877.

8 T. Guérin, O. Bénichou and R. Voituriez, Reactive conformations and non-Markovian cyclization kinetics of a Rouse polymer, J. Chem. Phys., 2013, 138(9), 094908.

9 T. Guérin, O. Bénichou and R. Voituriez, Non-Markovian polymer reaction kinetics, Nat. Chem., 2012, 4(7), 568-573.

10 A. Szabo, K. Schulten and Z. Schulten, First passage time approach to diffusion controlled reactions, J. Chem. Phys., 1980, 72(8), 4350-4357.

11 N. M. Toan, D. Marenduzzo, P. R. Cook and C. Micheletti, Depletion effects and loop formation in self-avoiding polymers, Phys. Rev. Lett., 2006, 97(17), 178302.

$12 \mathrm{~J}$. Shin and W. Sung, Effects of static and temporally fluctuating tensions on semiflexible polymer looping, $J$. Chem. Phys., 2012, 136(4), 045101.

13 M. Fritsche and D. W. Heermann, Confinement driven spatial organization of semiflexible ring polymers: implications for biopolymer packaging, Soft Matter, 2011, 7, 6906-6913.

14 J. Stampe and I. M. Sokolov, Cyclization of a polymer with charged reactive end groups, J. Chem. Phys., 2001, 114(11), 5043-5048. 
15 R. R. Cheng and D. E. Makarov, Failure of one-dimensional Smoluchowski diffusion models, J. Chem. Phys., 2011, 134(8), 085104.

16 N. M. Toan, G. Morrison, C. Hyeon and D. Thirumalai, Kinetics of loop formation in polymer chains, J. Phys. Chem. B, 2008, 112(19), 6094-6106.

17 K. V. Klenin and J. Langowski, Modeling of intramolecular reactions of polymers: an efficient method based on Brownian dynamics simulations, J. Chem. Phys., 2004, 121(10), 4951-4960.

18 J. S. Kim, V. Backman and I. Szleifer, Crowding-induced structural alterations of random-loop chromosome model, Phys. Rev. Lett., 2011, 106(16), 168102.

19 D. Marenduzzo, K. Finan and P. R. Cook, The depletion attraction: an under-appreciated force driving cellular organization, J. Cell Biol., 2006, 175(5), 681-686.

20 D. Marenduzzo, C. Micheletti and P. R. Cook, Entropydriven genome organization, Biophys. J., 2006, 90(10), 3712-3721.

21 H. X. Zhou, G. Rivas and A. P. Minton, Macromolecular crowding and confinement: biochemical, biophysical, and potential physiological consequences, Annu. Rev. Biophys., 2008, 37, 375-397.

22 C. Bräuchle, D. C. Lamb and J. Michaelis, SingleParticle Tracking and Single Molecule Energy Transfer, Wiley-VCH, Weinheim, Germany, 2012.

23 C. Micheletti, D. Marenduzzo and E. Orlandini, Polymers with spatial or topological constraints: theoretical and computational results, Phys. Rep., 2011, 504(1), 1-73.

24 J. Dorier and A. Stasiak, Modelling of crowded polymers elucidate effects of double-strand breaks in topological domains of bacterial chromosome, Nucleic Acids Res., 2013, 41(14), 6808-6815.

$25 \mathrm{~J}$. Shin, A. G. Cherstvy and R. Metzler, Mixing and segregation of ring polymers: spatial confinement and molecular crowding effects, New J. Phys., 2014, 16, 053047.

26 T. Cremer and M. Cremer, Chromosome territories, Cold Spring Harbor Perspect. Biol., 2010, 2(3), a003889.

27 T. B. Le, M. V. Imakaev, L. A. Mirny and M. T. Laub, Highresolution mapping of the spatial organization of a bacterial chromosome, Science, 2013, 342(6159), 731-734.

28 A. R. Denton, Crowding in polymer-nanoparticle mixtures, Int. Rev. Cell Mol. Biol., 2014, 307, 27-71.

29 F. Höfling and T. Franosch, Anomalous transport in the crowded world of biological cells, Rep Prog Phys, 2013, 76(4), 046602.

30 M. Weiss, Crowding, Diffusion, and Biochemical Reactions, Int. Rev. Cell Mol. Biol., 2014, 307, 383-417.

31 S. B. Zimmerman and A. P. Minton, Macromolecular crowding: biochemical, biophysical, and physiological consequences, Annu. Rev. Biophys. Biomol. Struct., 1993, 22(1), 27-65.

32 S. R. McGuffee and A. H. Elcock, Diffusion, crowding \& protein stability in a dynamic molecular model of the bacterial cytoplasm, PLoS Comput. Biol., 2010, 6(3), e1000694.

$33 \mathrm{~J}$. Szymanski and M. Weiss, Elucidating the origin of anomalous diffusion in crowded fluids, Phys. Rev. Lett., 2009, 103, 038102.
34 W. Pan, L. Filobelo, N. D. P. Pham, O. Galkin, V. V. Uzunova and P. G. Vekilov, Viscoelasticity in homogeneous protein solutions, Phys. Rev. Lett., 2009, 102, 058101.

35 C. K. Hu and M. S. Li, Dual effect of crowders on fibrillation kinetics of polypeptide chains revealed by lattice models, $J$. Chem. Phys., 2013, 138(18), 185101.

36 G. Schreiber, G. Haran and H. X. Zhou, Fundamental aspects of protein-protein association kinetics, Chem. Rev., 2009, 109(3), 839-860.

37 M. Erlkamp, S. Grobelny and R. Winter, Crowding effects on the temperature and pressure dependent structure, stability and folding kinetics of Staphylococcal Nuclease, Phys. Chem. Chem. Phys., 2014, 16(13), 5965-5976.

38 M. S. Cheung, D. Klimov and D. Thirumalai, Molecular crowding enhances native state stability and refolding rates of globular proteins, Proc. Natl. Acad. Sci. U. S. A., 2005, 102(13), 4753-4758.

39 C. Hyeon and D. Thirumalai, Capturing the essence of folding and functions of biomolecules using coarsegrained models, Nat. Commun., 2011, 2, 487.

40 M. Guo, Y. Xu and M. Gruebele, Temperature dependence of protein folding kinetics in living cells, Proc. Natl. Acad. Sci. U. S. A., 2012, 109(44), 17863-17867.

41 M. del Alamo, G. Rivas and M. G. Mateu, Effect of macromolecular crowding agents on human immunodeficiency virus type 1 capsid protein assembly in vitro, J. Virol., 2005, 79(22), 14271-14281.

42 N. A. Denesyuk and D. Thirumalai, Crowding promotes the switch from hairpin to pseudoknot conformation in human telomerase RNA, J. Am. Chem. Soc., 2011, 133(31), 1185811861.

43 A. V. Weigel, B. Simon, M. M. Tamkun and D. Krapf, Ergodic and nonergodic processes coexist in the plasma membrane as observed by single-molecule tracking, Proc. Natl. Acad. Sci. U. S. A., 2011, 108(16), 6438-6443.

44 J. H. Jeon, H. M. S. Monne, M. Javanainen and R. Metzler, Anomalous diffusion of phospholipids and cholesterols in lipid bilayer and its origins, Phys. Rev. Lett., 2012, 109, 188103.

45 M. Javanainen, H. Hammaren, L. Monticelli, J. H. Jeon, R. Metzler and I. Vattulainen, Anomalous and normal diffusion of proteins and lipids in crowded lipid membranes, Faraday Discuss., 2013, 161, 397-417.

46 N. A. Mahynski, B. Irick and A. Z. Panagiotopoulos, Structure of phase-separated athermal colloid-polymer systems in the protein limit, Phys. Rev. E: Stat., Nonlinear, Soft Matter Phys., 2013, 87, 022309.

47 P. Paricaud, S. Varga and G. Jackson, Study of the demixing transition in model athermal mixtures of colloids and flexible self-excluding polymers using the thermodynamic perturbation theory of Wertheim, J. Chem. Phys., 2003, 118(8), 8525-8536.

48 D. Swigon, B. D. Coleman and W. K. Olson, Modeling the Lac repressor-operator assembly: the influence of DNA looping on Lac repressor conformation, Proc. Natl. Acad. Sci. U. S. A., 2006, 103(26), 9879-9884.

49 D. G. Priest, L. Cu, S. Kumar, D. D. Dunlap, I. B. Dodd and K. E. Shearwin, Quantitation of the DNA tethering effect in 
long-range DNA looping in vivo and in vitro using the Lac and $\lambda$ repressors, Proc. Natl. Acad. Sci. U. S. A., 2014, 111(1), 349-354.

50 Z. Hensel, X. Weng, A. C. Lagda and J. Xiao, Transcriptionfactor-mediated DNA looping probed by high-resolution, single-molecule imaging in live E. coli cells, PLoS Biol., 2013, 11(6), e1001591.

51 B. van den Broek, M. A. Lomholt, S. M. Kalisch, R. Metzler and G. J. Wuite, How DNA coiling enhances target localization by proteins, Proc. Natl. Acad. Sci. U. S. A., 2008, 105(41), 15738-15742.

52 M. A. Lomholt, B. van den Broek, S. M. J. Kalisch, G. J. L. Wuite and R. Metzler, Facilitated diffusion with DNA coiling, Proc. Natl. Acad. Sci. U. S. A., 2009, 106(20), 8204-8208.

53 P. R. ten Wolde and A. Mugler, Importance of crowding in signaling, genetic, and metabolic networks, Int. Rev. Cell Mol. Biol., 2013, 307, 419-442.

54 M. Tabaka, T. Kalwarczyk and R. Holyst, Quantitative influence of macromolecular crowding on gene regulation kinetics, Nucleic Acids Res., 2014, 42(2), 727-738.

55 A. G. Cherstvy and V. B. Teif, "Structure-driven homology pairing of chromatin fibers: the role of electrostatics and protein-induced bridging”, J. Biol. Phys., 2013, 39(3), 363385.

56 H. X. Zhou, Protein folding in confined and crowded environments, Arch. Biochem. Biophys., 2008, 469(1), 76-82.

57 N. F. Dupuis, E. D. Holmstrom and D. J. Nesbitt, Molecularcrowding effects on single-molecule RNA folding/unfolding thermodynamics and kinetics, Proc. Natl. Acad. Sci. U. S. A., 2014, 111(23), 8464-8469.

58 G. Bonnet, O. Krichevsky and A. Libchaber, Kinetics of conformational fluctuations in DNA hairpin-loops, Proc. Natl. Acad. Sci. U. S. A., 1998, 95(15), 8602-8606.

59 R. Frederickx, T. in't Veld and E. Carlon, Anomalous Dynamics of DNA Hairpin Folding, Phys. Rev. Lett., 2014, 112, 198102.

60 O. Stiehl, K. Weidner-Hertrampf and M. Weiss, Kinetics of conformational fluctuations in DNA hairpin-loops in crowded fluids, New J. Phys., 2013, 15(11), 113010.

61 B. Lu and A. R. Denton, Crowding of polymer coils and demixing in nanoparticle-polymer mixtures, J. Phys.: Condens. Matter, 2011, 23(28), 285102.

62 A. Kudlay, C. Hyeon and D. Thirumalai, Influence of the Shape of Crowding Particles on the Structural Transitions in a Polymer, J. Phys. Chem. B, 2012, 116(29), 8513-8522.

63 A. R. Denton and M. Schmidt, Mixtures of charged colloid and neutral polymer: Influence of electrostatic interactions on demixing and interfacial tension, J. Chem. Phys., 2005, 122(24), 244911.

64 A. R. Denton, Crowding in polymer-nanoparticle mixtures, Int. Rev. Cell Mol. Biol., 2014, 307, 27-71.

65 N. K. Lee, C. F. Abrams and A. Johner, Optimal confinement for internal polymer binding, EPL, 2005, 72(6), 922-928.

66 C. F. Abrams, N. K. Lee and A. Johner, Diffusion-reaction in confined polymer chains, Macromolecules, 2006, 39(10), 3655-3663.
67 K. Kremer and G. S. Grest, Dynamics of entangled linear polymer melts: A molecular dynamics simulation, $J$. Chem. Phys., 1990, 92, 5057-5086.

68 P. Bhattacharyya, R. Sharma and B. J. Cherayil, Confinement and viscoelastic effects on chain closure dynamics, J. Chem. Phys., 2012, 136(23), 234903; compare also R. Chakrabarty, Physica A, 2014, 391, 5326-5331.

69 J. Shin, A. G. Cherstvy and R. Metzler, Sensing viruses by mechanical tension of DNA in responsive hydrogels, Phys. Rev. X, 2014, 4, 021002.

70 D. Shore, J. Langowski and R. L. Baldwin, DNA flexibility studied by covalent closure of short fragments into circles, Proc. Natl. Acad. Sci. U. S. A., 1981, 78(8), 48334837.

71 A. Vologodskii and M. D. Frank-Kamenetskii, Strong bending of the DNA double helix, Nucleic Acids Res., 2013, 41(14), 6785-6792.

72 N. A. Mahynski, A. Z. Panagiotopoulos, D. Meng and S. K. Kumar, Stabilizing colloidal crystals by leveraging void distributions, Nat. Commun., 2014, 5, 4472.

73 D. Jost and R. Everaers, A Unified Poland-Scheraga Model of Oligo-and Polynucleotide DNA Melting: Salt Effects and Predictive Power, Biophys. J., 2009, 96(3), 1056-1067.

74 E. Zaccarelli and W. C. K. Poon, Colloidal glasses and gels: The interplay of bonding and caging, Proc. Natl. Acad. Sci. U. S. A., 2009, 106(36), 15203-15208.

75 J. SantaLucia Jr, A unified view of polymer, dumbbell, and oligonucleotide DNA nearest-neighbor thermodynamics, Proc. Natl. Acad. Sci. U. S. A., 1998, 95(4), 1460-1465.

76 Y. Chen and K. Luo, Dynamics of polymer translocation through a nanopore induced by different sizes of crowding agents, J. Chem. Phys., 2013, 138(20), 204903.

77 A. G. Cherstvy, DNA cyclization: suppression or enhancement by electrostatic repulsions?, J. Phys. Chem. B, 2011, 115(15), 4286-4294.

78 S. I. Nakano, D. Miyoshi and N. Sugimoto, Effects of Molecular Crowding on the Structures, Interactions, and Functions of Nucleic Acids, Chem. Rev., 2013, 114(5), 2733-2758.

79 T. Ando, E. Chow and J. Skolnick, Dynamic simulation of concentrated macromolecular solutions with screened long-range hydrodynamic interactions: algorithm and limitations, J. Chem. Phys., 2013, 139(12), 121922.

80 R. G. Winkler, Analytical calculation of the relaxation dynamics of partially stretched flexible chain molecules: necessity of a worm-like chain description, Phys. Rev. Lett., 1999, 82(9), 1843-1846.

81 R. G. Winkler, S. Keller and J. O. Rädler, Intramolecular dynamics of linear macromolecules by fluorescence correlation spectroscopy, Phys. Rev. E: Stat., Nonlinear, Soft Matter Phys., 2006, 73(4), 041919.

82 R. Shusterman, S. Alon, T. Gavrinyov and O. Krichevsky, Monomer Dynamics in Double- and Single-Stranded DNA Polymers, Phys. Rev. Lett., 2004, 92(4), 048303.

83 E. P. Petrov, T. Ohrt, R. G. Winkler and R. Schwille, Diffusion and segmental dynamics of double-stranded DNA, Phys. Rev. Lett., 2006, 97(25), 258101. 
84 D. E. Smith and S. Chu, Response of Flexible Polymers to a Sudden Elongational Flow, Science, 1998, 281(5381), 13351340.

85 T. T. Perkins, S. R. Quake, D. E. Smith and S. Chu, Relaxation of a singleDNA molecule observed by optical microscopy, Science, 1994, 264(5160), 822-826.

86 Y. R. Chemla and T. Ha, Ultraslow relaxation of confined DNA, Science, 2014, 345(6195), 380-381.

87 Y. von Hansen, M. Hinczewski and R. R. Netz, Hydrodynamic screening near planar boundaries: Effects on semiflexible polymer dynamics, J. Chem. Phys., 2011, 134, 235102.

88 G. Schreiber, G. Haran and H. X. Zhou, Fundamental aspects of protein-protein association kinetics, Chem. Rev., 2009, 109(3), 839-860.

89 J. Shin, A. G. Cherstvy and R. Metzler, work in progress.

90 A. Y. Grosberg and A. R. Khokhlov, Statistical Physics of Macromolecules, AIP Press, Woodbury, NY, 1994.

91 X. Li and A. B. Kolomeisky, Mechanisms and topology determination of complex chemical and biological network systems from first-passage theoretical approach, J. Chem. Phys., 2013, 139(14), 144106.

92 A. Valleriani, X. Li and A. B. Kolomeisky, Unveiling the hidden structure of complex stochastic biochemical networks, J. Chem. Phys., 2014, 140(6), 064101.

93 H. S. Chung, J. M. Louis and W. A. Eaton, Experimental determination of upper bound for transition path times in protein folding from single-molecule photon-by-photon trajectories, Proc. Natl. Acad. Sci. U. S. A., 2009, 106(29), 11837-11844.

94 K. Neupane, D. B. Ritchie, H. Yu, D. A. Foster, F. Wang and M. T. Woodside, Transition path times for nucleic acid folding determined from energy-landscape analysis of single-molecule trajectories, Phys. Rev. Lett., 2012, 109(6), 068102.

95 K. W. Minton, P. Karmin, G. M. Hahn and A. P. Minton, Nonspecific stabilization of stress-susceptible proteins by stress-resistant proteins, Proc. Natl. Acad. Sci. U. S. A., 1982, 79(23), 7107-7111.

96 N. G. Van Kampen, Stochastic Processes in Physics and Chemistry, North Holland, 3rd edn, 2007.

97 S. Mukherjee, M. M. Waegele, P. Chowdhury, L. Guo and F. Gai, Effect of macromolecular crowding on protein folding dynamics at the secondary structure level, J. Mol. Biol., 2009, 393(1), 227-236.

98 R. Metzler and J. Klafter, The random walk's guide to anomalous diffusion: a fractional dynamics approach, Phys. Rep., 2000, 339(1), 1-77.
99 E. Barkai, Y. Garini and R. Metzler, Strange kinetics of single molecules in living cells, Phys. Today, 2012, 65(8), 29-35.

100 R. Metzler and J. Klafter, The restaurant at the end of the random walk: recent developments in the description of anomalous transport by fractional dynamics, J. Phys. A: Math. Gen., 2004, 37(31), R161-R208.

101 R. Metzler, J. H. Jeon, A. G. Cherstvy and E. Barkai, Anomalous diffusion models and their properties: nonstationarity, non-ergodicity, and ageing at the centenary of single particle tracking, Phys. Chem. Chem. Phys., 2014, 16, 24128-24164.

102 A. B. Goins, H. Sanabria and M. N. Waxham, Macromolecular Crowding and Size Effects on Probe Microviscosity, Biophys. J., 2008, 95(11), 5362-5373.

103 A. P. Minton, Hard Quasi-spherical Particle Models for the Viscosity of Solutions of Protein Mixtures, J. Phys. Chem. B, 2012, 116(31), 9310-9315.

104 S. V. Kuznetsov, Y. Shen, A. S. Benight and A. Ansari, A Semiflexible Polymer Model Applied to Loop Formation in DNA Hairpins, Biophys. J., 2001, 81(5), 2864-2875.

105 M. T. Woodside, et al. Nanomechanical measurements of the sequence-dependent folding landscapes of single nucleic acid hairpins, Proc. Natl. Acad. Sci. U. S. A., 2005, 103(16), 6190-6195.

106 Microcompartmentation and Phase Separation in Cytoplasm, A Survey of Cell Biology, ed. H. Walter, D. Brooks and P. Srere, 1999, Intl. Rev. Cell \& Mol. Biol. 192.

107 S. K. Ghosh, A. G. Cherstvy, R. Metzler, work in progress.

108 C. A. Strulson, et al., RNA catalysis through compartmentalization, Nat. Chem., 2012, 4, 941-946.

$109 \mathrm{H}$. Walter and D. E. Brooks, Phase separation in cytoplasm, due to macromolecular crowding, is the basis for microcompartmentation, FEBS Lett., 1995, 361, 135-139.

110 N. F. Dupuis, E. D. Holmstrom and D. J. Nesbitt, Molecularcrowding effects on single-molecule RNA folding/unfolding thermodynamics and kinetics, Proc. Natl. Acad. Sci. U. S. A., 2014, 111(23), 8464-8469.

111 H. Kang, P. A. Pincus, C. Hyeon and D. Thirumalai, 2014, Effects of macromolecular crowding on the collapse of biopolymers, arXiv:1409.5663.

112 I. Oh, S. Choi, Y. J. Jung and J. S. Kim, Unusual sizedependence of effective interactions between collapsed polymers in crowded environments, Soft Matter, 2014, 10, 9098-9104.

113 T. N. Shendruk et al., Simulating the Entropic Collapse of Coarse-Grained Chromosomes, 2014, arXiv:1407.2850. 